\title{
LHCb Physics and 2010-11 prospects
}

Stefano Perazzini

On behalf of LHCb Collaboration

MENU2010

$2^{\text {nd }}$ June 2010 


\section{OUTLINE}

- Physics:

- Flavor physics and CPV in the quark sector

- Search for New Physics

- The LHCb Experiment

- The detector

- Data taking in 2010-11 run

- Core analyses and prospects for 2010-11 


\section{Flavor physics in quark sector}

$$
L_{\text {int }}^{C C}=-\frac{g}{\sqrt{2}}\left(\begin{array}{lll}
\bar{u} & \bar{c} & \bar{t}
\end{array}\right) \gamma^{\mu} V_{C K M}\left(\begin{array}{l}
d \\
s \\
b
\end{array}\right)+h c . \quad V_{C K M}=\left(\begin{array}{lll}
V_{u d} & V_{u s} & V_{u b} \\
V_{c d} & V_{c s} & V_{c b} \\
V_{t d} & V_{t s} & V_{t b}
\end{array}\right)
$$

- Interacting (flavor) eigenstates are mixtures of mass eigenstates described in the SM by the CKM matrix

- New particles in loop diagrams may modify measured quantities related to $V_{C K M}$ elements
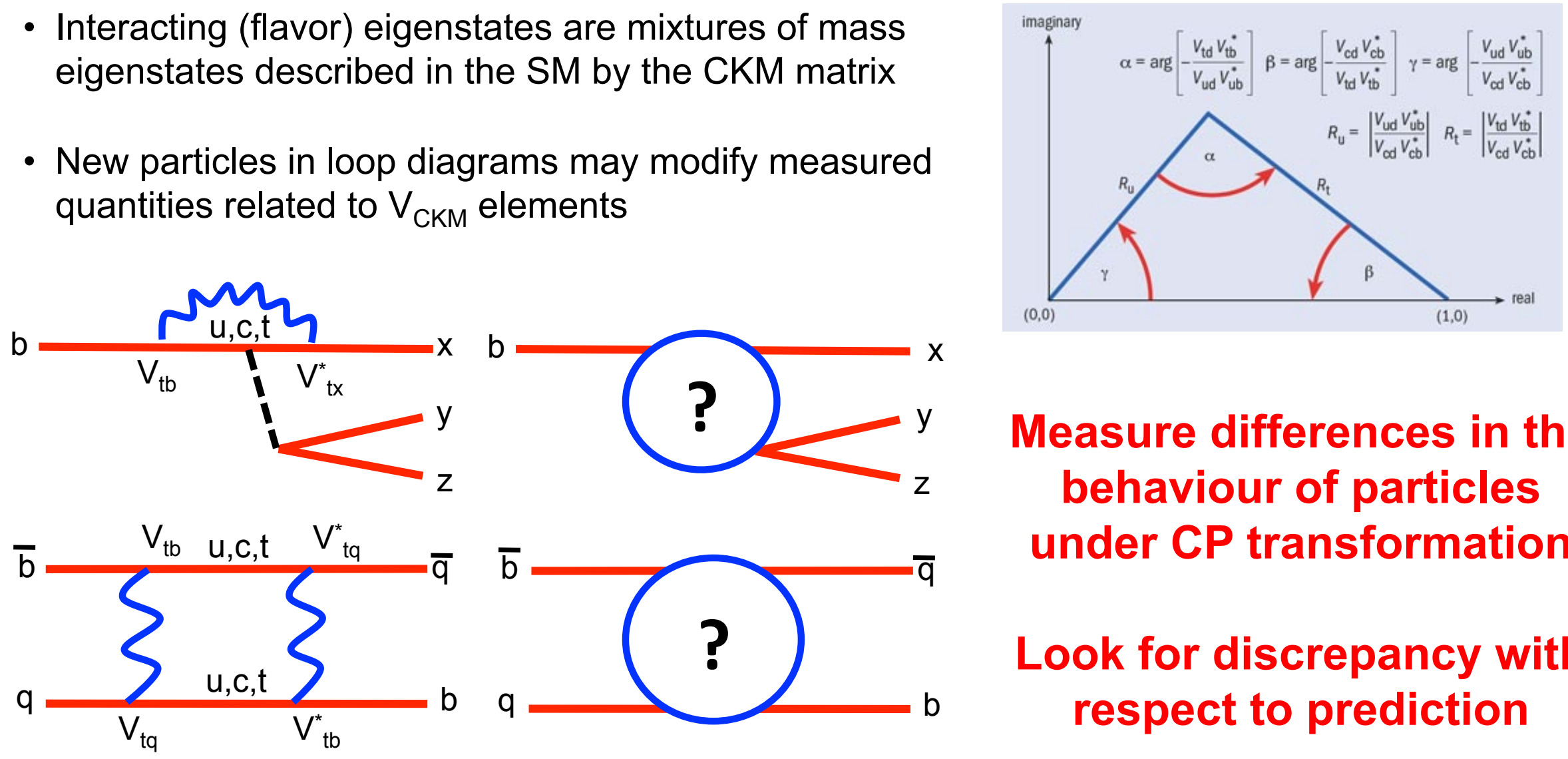

Measure differences in the behaviour of particles under CP transformation

Look for discrepancy with respect to prediction 


\section{Status of the Art}

- Good agreement from all measurements

- Still open windows to NP corrections at $10-20 \%$

- $\sigma(\gamma) \sim 15-20^{\circ}$

- $\mathrm{B}_{\mathrm{s}}$ mixing phase

- Rare decays:

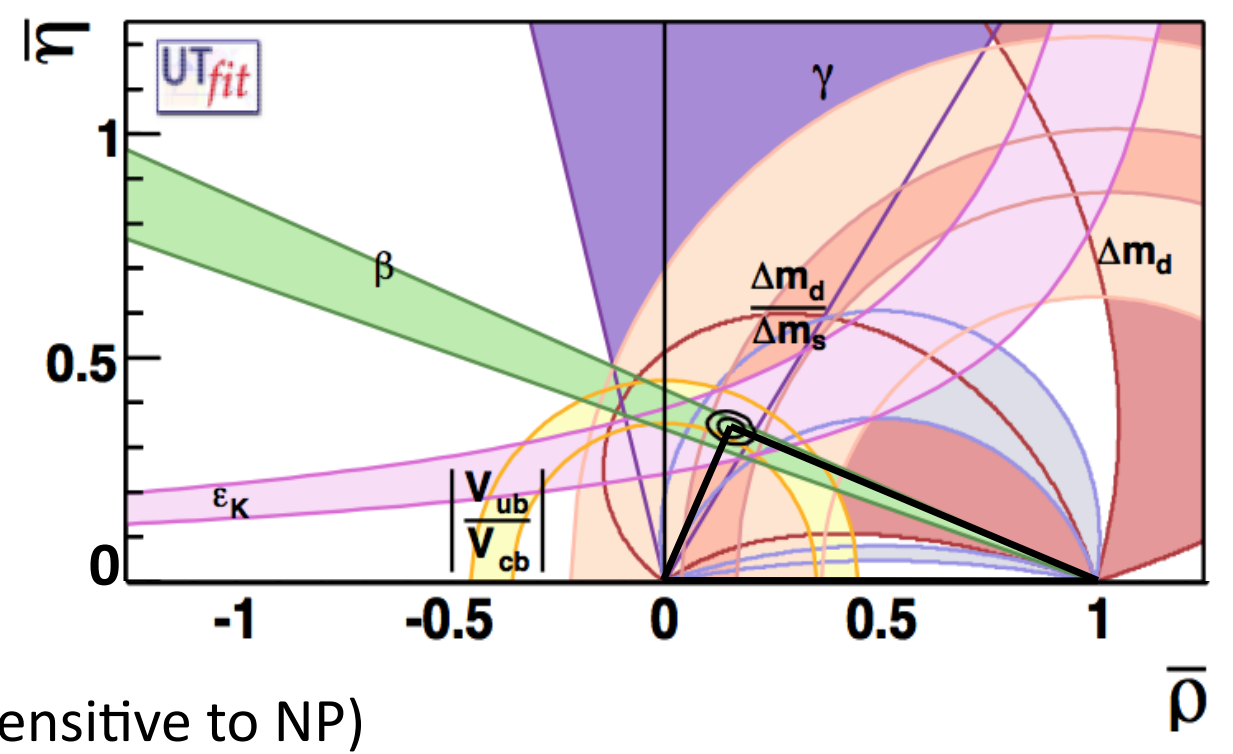

- $B_{s} \rightarrow \mu^{+} \mu^{-}:$BR not measured (sensitive to NP)

- $B_{d} \rightarrow K^{*} \mu^{+} \mu^{-}$: not clear measurements status

- Charm sector: mixing induced CP-violation needs more precise measurements 


\section{LHCb Detector}

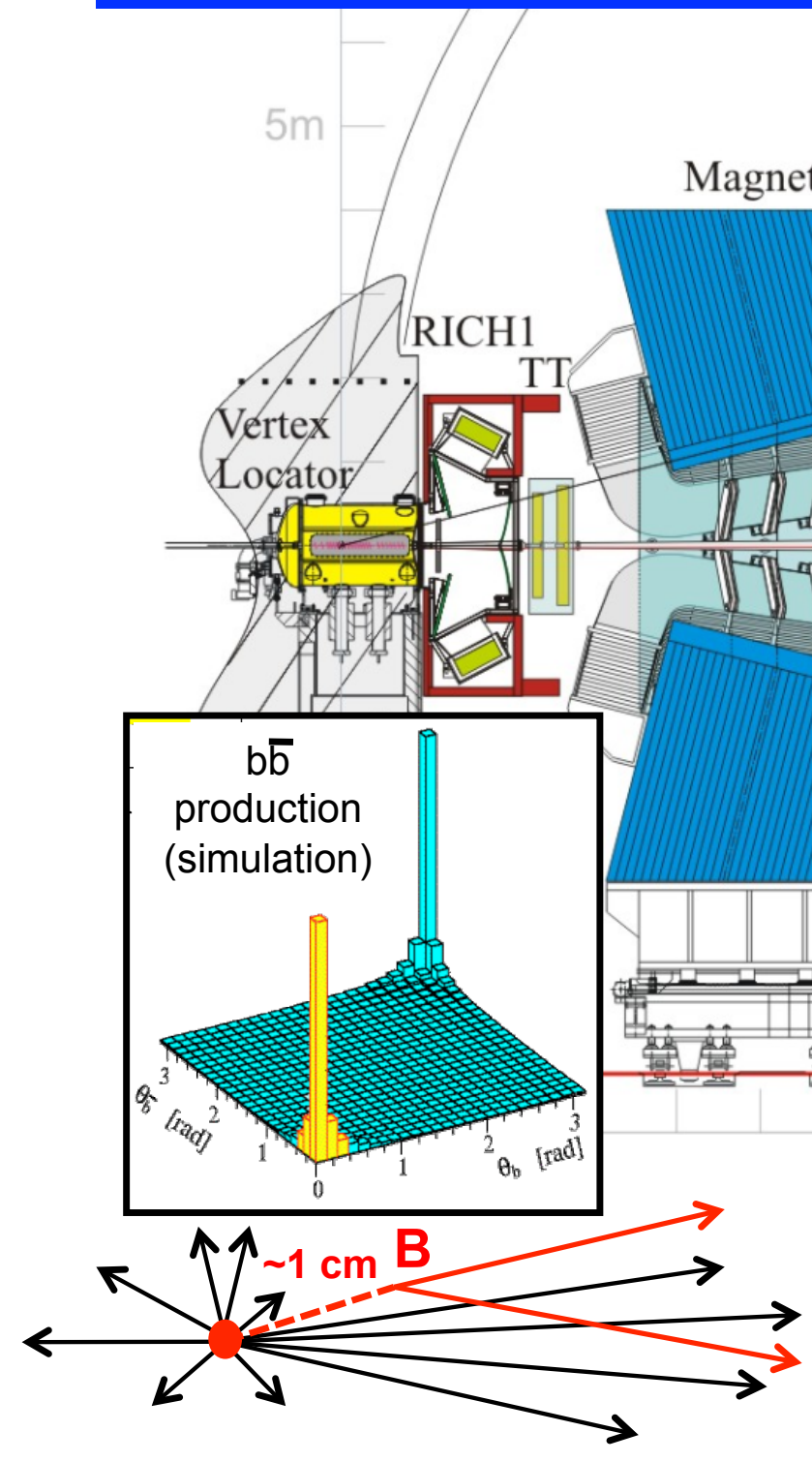

02-06-2010

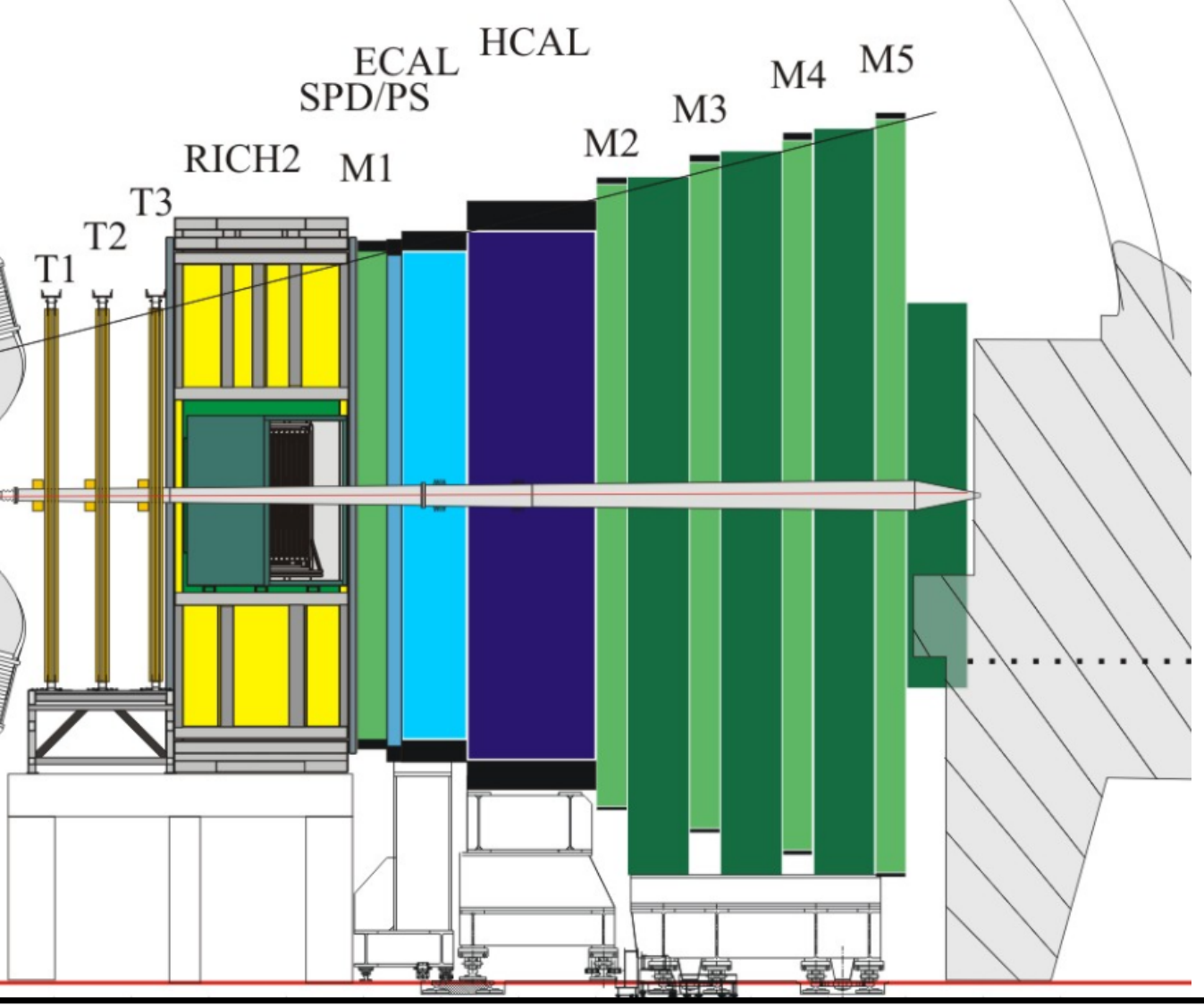

- Excellent vertexing (high proper time resolution)

- Excellent tracking (invariant mass resolution)

- Particle ID (discrimination of various hadrons)

- Trigger on high $\mathrm{E}_{\mathrm{T}}$ hadrons and high $\mathrm{p}_{\mathrm{T}}$ muons (hints of $B$ presence) 


\section{Detector status (I)}
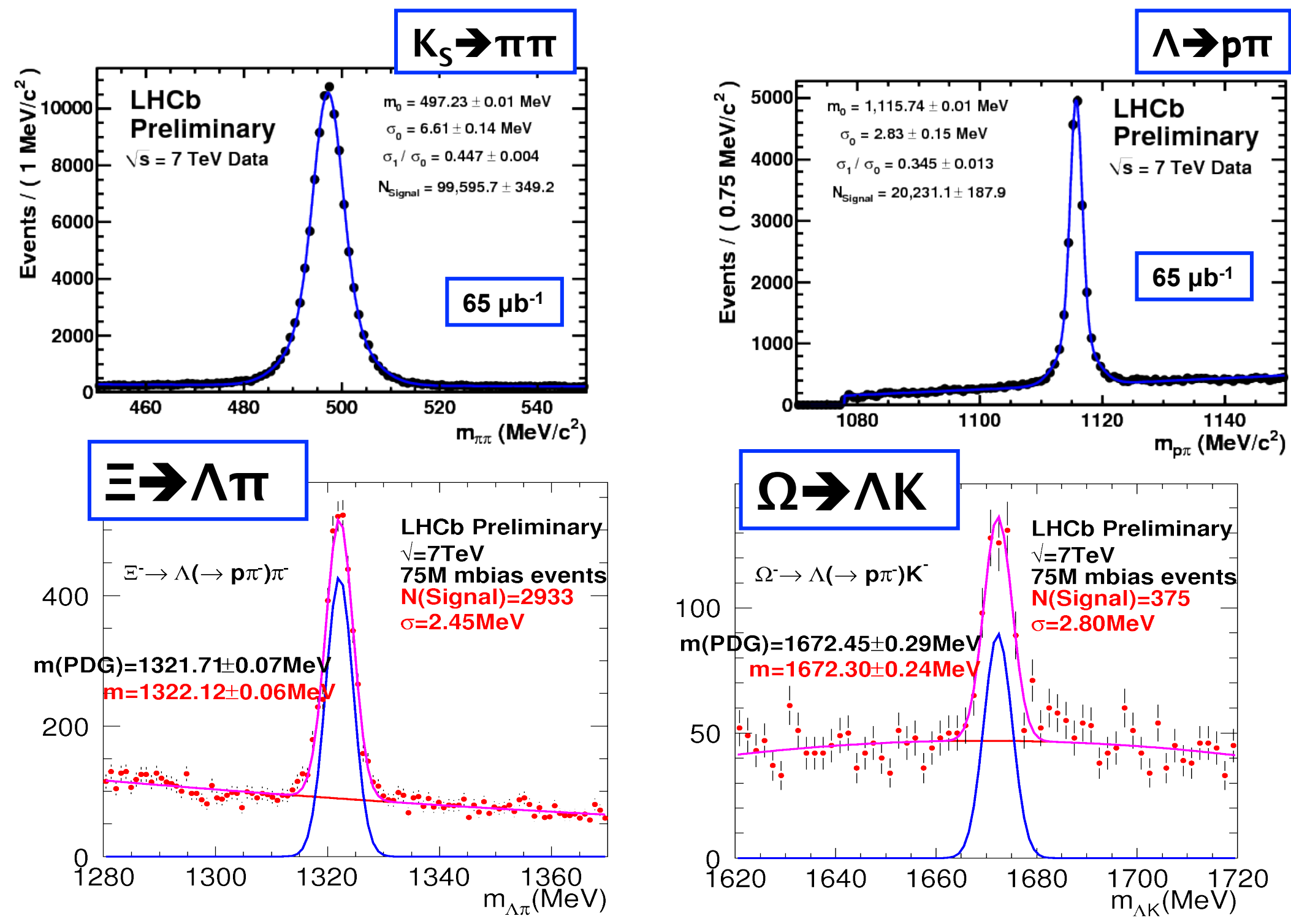


\section{Detector status (II)}
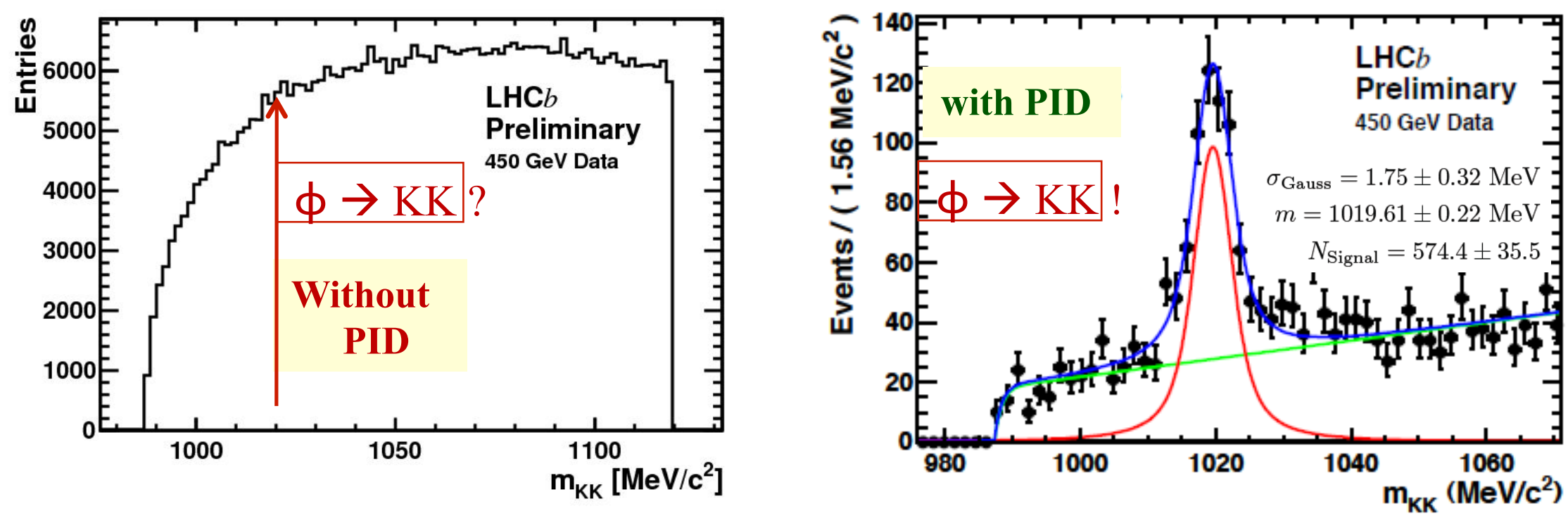

$\mathrm{m}(\mathrm{pdg})=1019.455 \pm 0.020 \mathrm{MeV}$

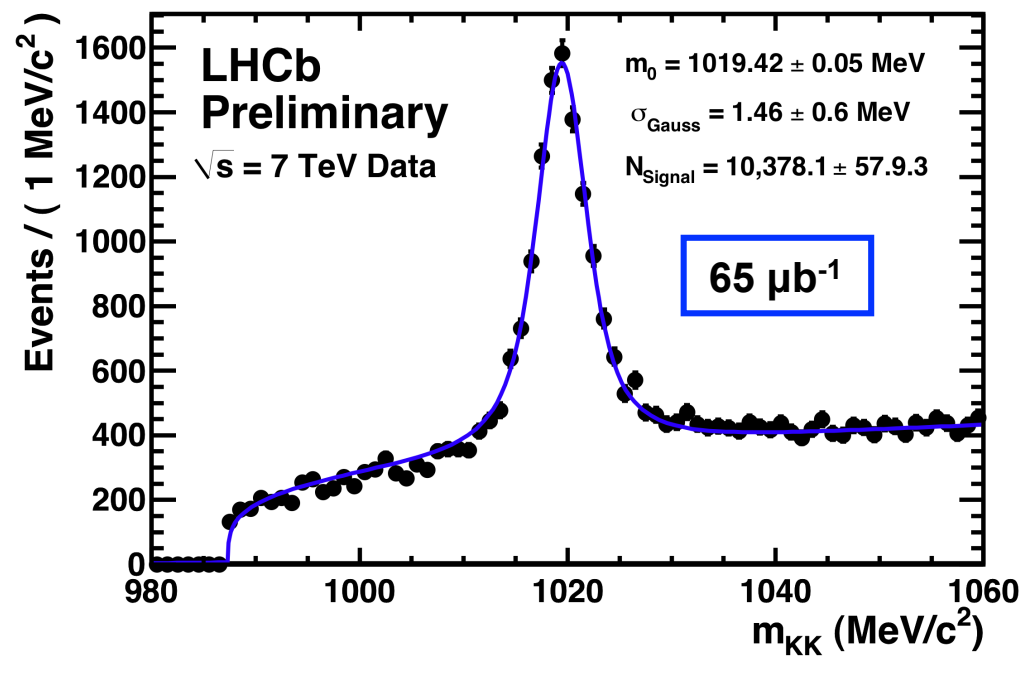

\section{Peak seen cutting only using Particle ID}




\title{
2010-11 Data Taking
}

\begin{tabular}{|c|c|c|c|}
\hline \multicolumn{4}{|c|}{ Assumed conditions in MC studies pre-2010 } \\
\hline Vs & $\sigma_{\mathrm{bb}}$ & $L$ & $\begin{array}{c}1 \text { year integrated } \\
\text { luminosity }\end{array}$ \\
\hline $14 \mathrm{TeV}$ & $500 \mu \mathrm{b}$ & $2 \times 10^{32} \mathrm{~cm}^{-2} \mathrm{~s}^{-1}$ & $2 \mathrm{fb}^{-1}$ \\
\hline
\end{tabular}

\author{
Mainwork about LHCb key \\ measurements \\ (arXiv:0912.4179v2 [hep-ex])
}
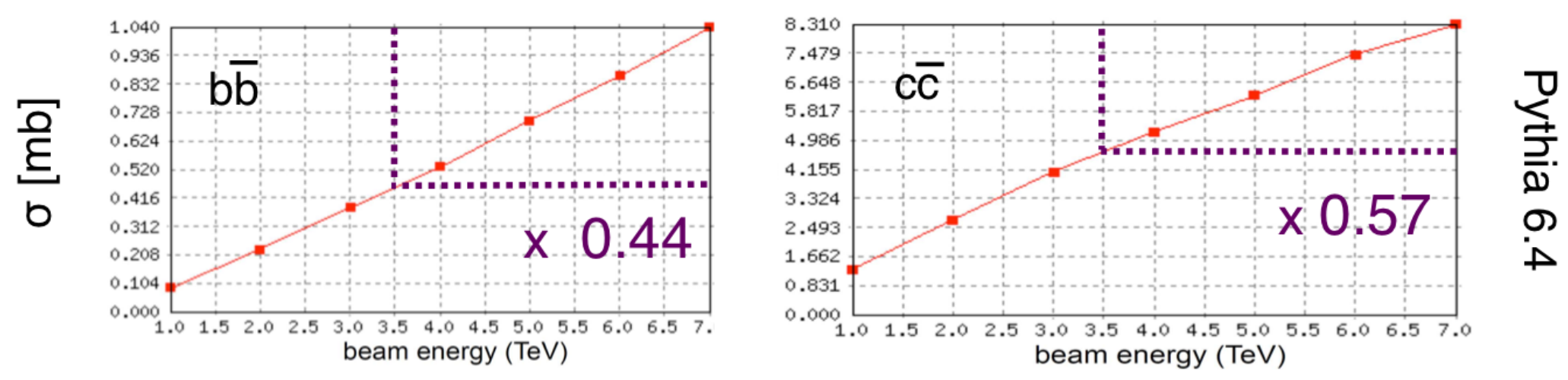

\begin{tabular}{|c|c|c|c|}
\hline \multicolumn{4}{|c|}{2010 expected conditions } \\
\hline$\sqrt{ } \mathrm{s}$ & $\sigma_{\mathrm{bb}}$ & $\sigma_{\mathrm{cc}}$ & $L$ \\
\hline $7 \mathrm{TeV}$ & $500 \mu \mathrm{b}$ & $4.7 \mathrm{mb}$ & $<2 \times 10^{31} \mathrm{~cm}^{-2} \mathrm{~s}^{-1}$ \\
\hline
\end{tabular}

- Some loss in signal yield due to $\sqrt{ } s=7 \mathrm{TeV}$

- Release of trigger thresholds

- $\varepsilon_{\text {trig }}^{\text {charm } ~} 40-50 \%$

- Expected $0.1 \mathrm{fb}^{-1}$ of integrated luminosity

\begin{tabular}{|c|c|c|c|}
\hline \multicolumn{4}{|c|}{2011 expected conditions } \\
\hline$\sqrt{ } \mathrm{s}$ & $\sigma_{\mathrm{bb}}$ & $\sigma_{\mathrm{cc}}$ & $L$ \\
\hline $7 \mathrm{TeV}$ & $500 \mu \mathrm{b}$ & $4.7 \mathrm{mb}$ & $\sim 10^{32} \mathrm{~cm}^{-2} \mathrm{~s}^{-1}$ \\
\hline
\end{tabular}

- $\angle$ close to design value

- $\varepsilon_{\text {trig }}$ charm $\sim 10 \%$

- $\varepsilon_{\text {trig }} \mathrm{B} \sim 75-80 \%$

- $\varepsilon_{\text {trig }} B \rightarrow \mu X>90 \%$

- Expected $1 \mathrm{fb}^{-1}$ of integrated luminosity 


\section{$\mathrm{b} \overline{\mathrm{b}}$ production}

- Evaluation of $\sigma_{\mathrm{bb}}$ from ratio between prompt and displaced $\mathrm{J} / \Psi$

- Combination of invariant mass and pseudo proper-time fits
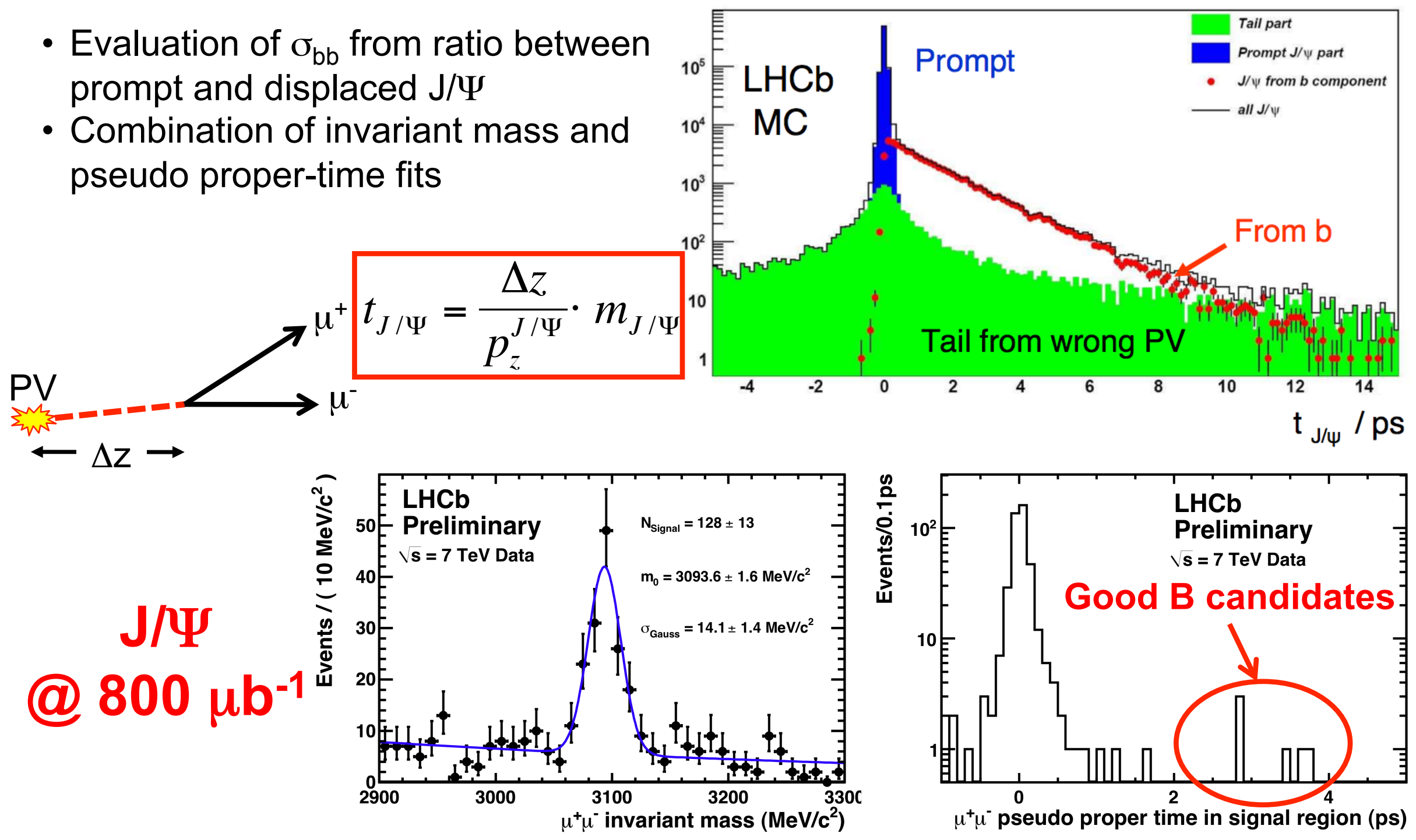


\section{$\mathrm{J} / \Psi$ production}

- $\mathrm{J} / \Psi$ is also important by itself:

- All models nowadays fail to incorporate cross section and polarization

- No much data at high pseudorapidity

- $\mathrm{J} / \Psi$ also produced in decay of various charmonium states
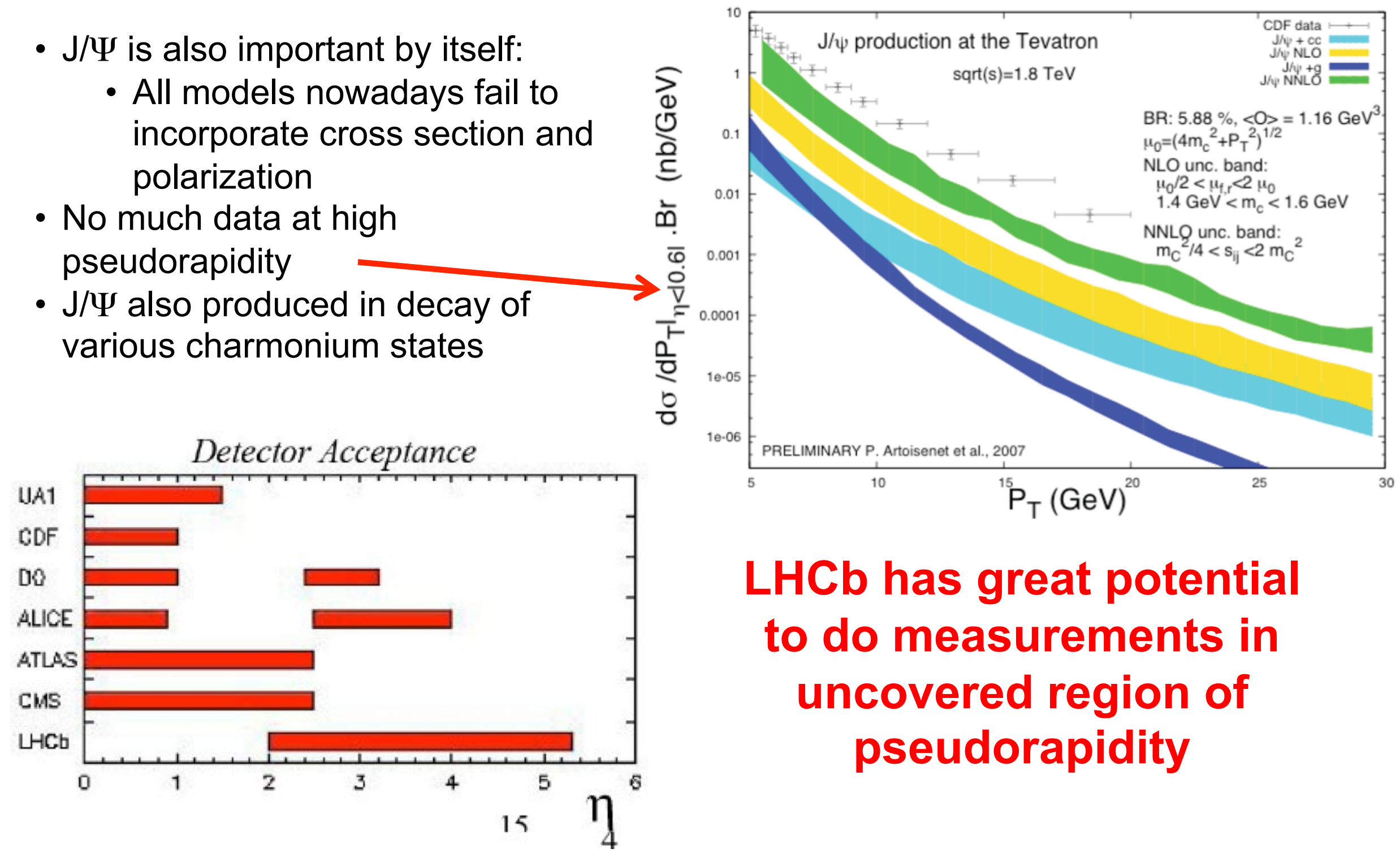


\section{Mixing and CPV in charm}

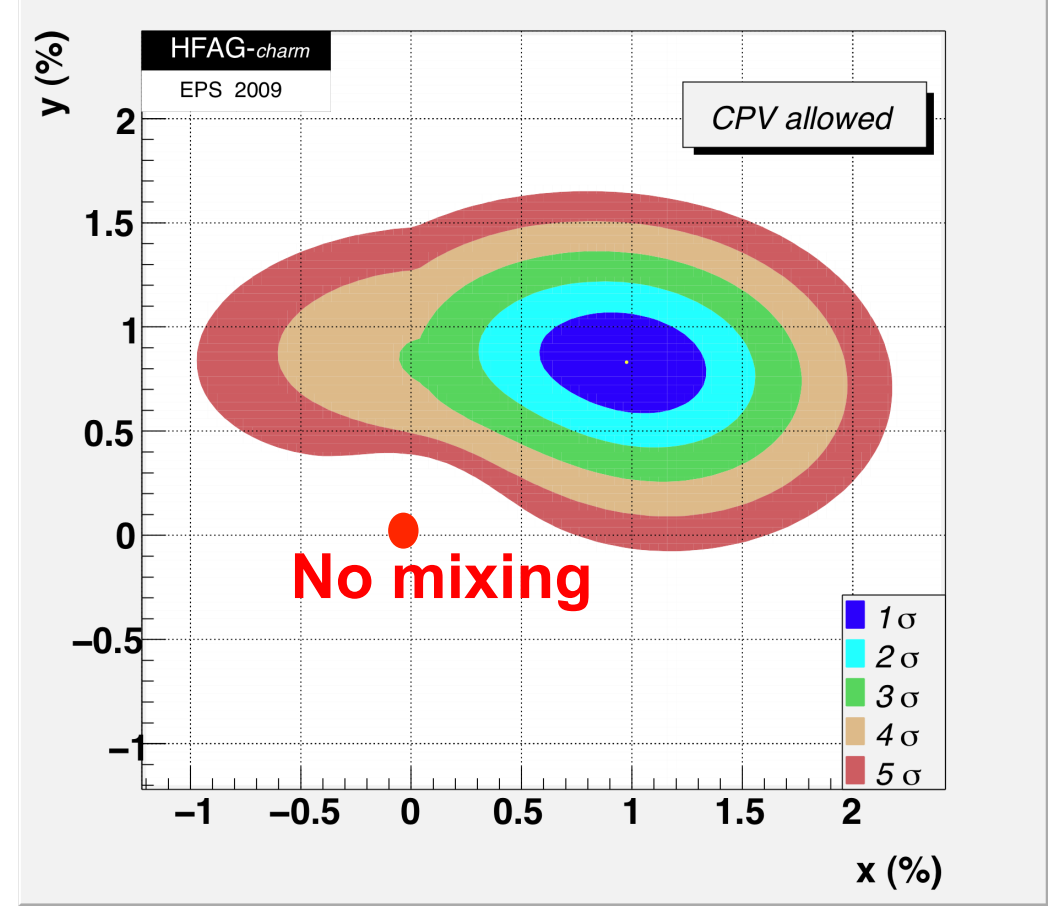

- Neutral D mixing is nowadays a matter of facts

- More precise measurements needed for CPV programme

- CPV negligible in the SM

- window for NP discovery

- $x$ and $y$ affect precision on CPV measurements

$$
A_{C P} \sim A_{M} y \cos \Phi-x \sin \Phi
$$

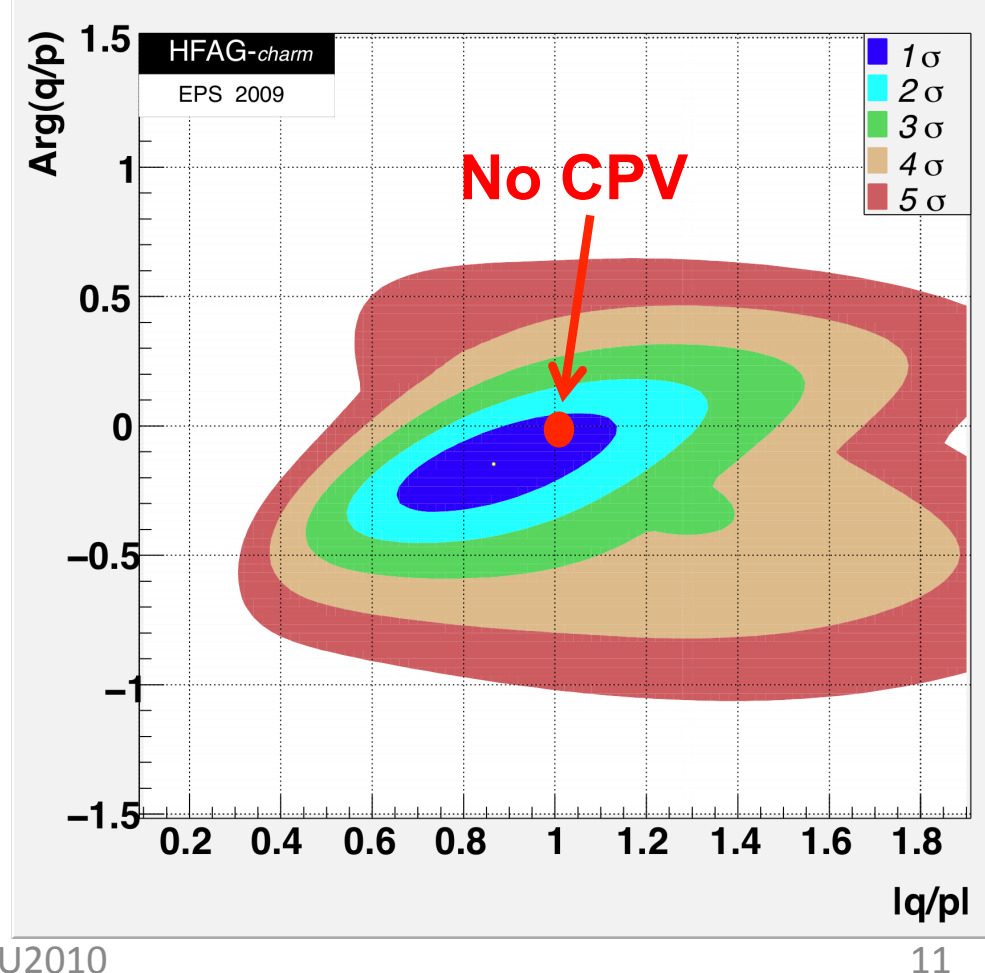




\section{Mixing and CPV in charm}

1. Measure " $\mathrm{y}_{\mathrm{cp}}$ ", that means y modified by CPV effects $2.7 \times 10^{6}$ untagged $\mathrm{K} \pi \quad 2.6 \times 10^{5}$ untagged $\mathrm{KK}$

$$
\begin{aligned}
& y_{C P}=\frac{\tau\left(D^{0} \rightarrow K^{-} \pi^{+}\right)}{\tau\left(D^{0} \rightarrow h^{+} h^{-}\right)}-1 \\
& y_{C P}=y \cos \Phi_{D}-\left(\left|\frac{q}{p}\right|^{2}-1\right) x \sin \Phi_{D}
\end{aligned}
$$

Measure of $\mathrm{A}_{\Gamma}$ :

$A_{\Gamma}(t)=\frac{\tau\left(\bar{D}^{0} \rightarrow h^{+} h^{-}\right)-\tau\left(D^{0} \rightarrow h^{+} h^{-}\right)}{\tau\left(\bar{D}^{0} \rightarrow h^{+} h^{-}\right)+\tau\left(D^{0} \rightarrow h^{+} h^{-}\right)}$

$A_{\Gamma}=\left(\left|\frac{q}{p}\right|^{2}-1\right) y \cos \Phi_{D}-x \sin \Phi_{D}$

\section{$1.1 \times 10^{5}$} tagged $D^{*} \rightarrow D^{0}(\rightarrow K K) \pi$

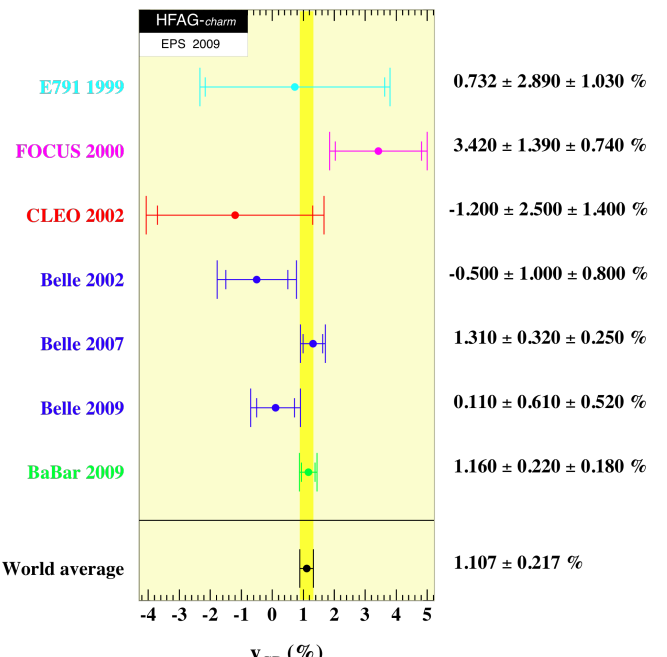

$$
\mathbf{y}_{\mathrm{CP}}(\%)
$$

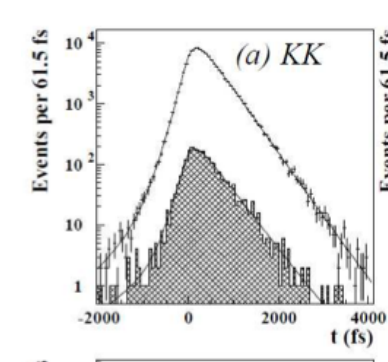

$$
1 \text { 年 }
$$

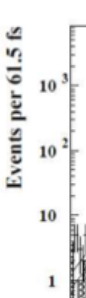
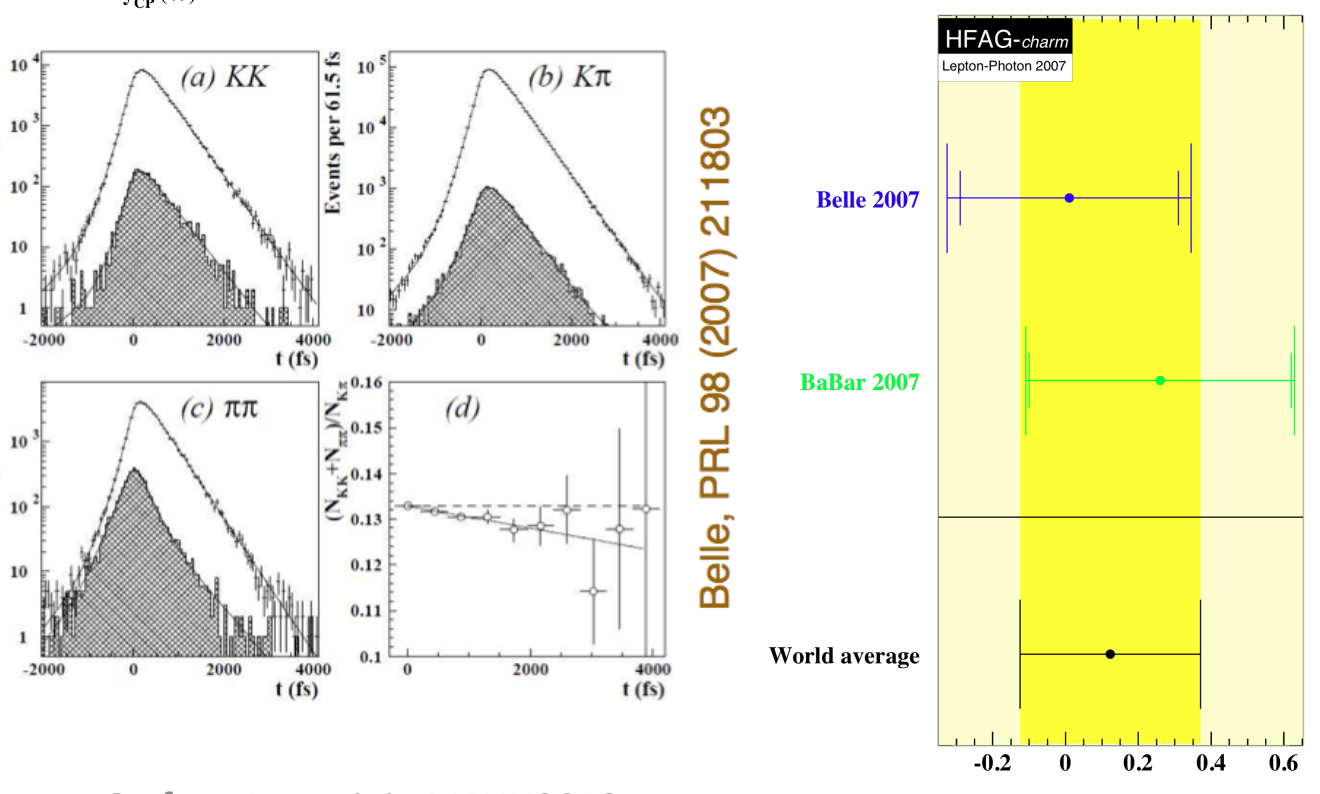

$\mathbf{A}_{\Gamma}(\%)$
$0.010 \pm 0.300 \pm 0.150 \%$

$0.260 \pm 0.360 \pm 0.080 \%$

$0.123 \pm 0.248 \%$ 


\section{$\mathrm{D}^{0}$ in LHCb @ 800ub-1}

$\mathrm{D}^{0} \rightarrow \mathrm{K} \pi$ untagged

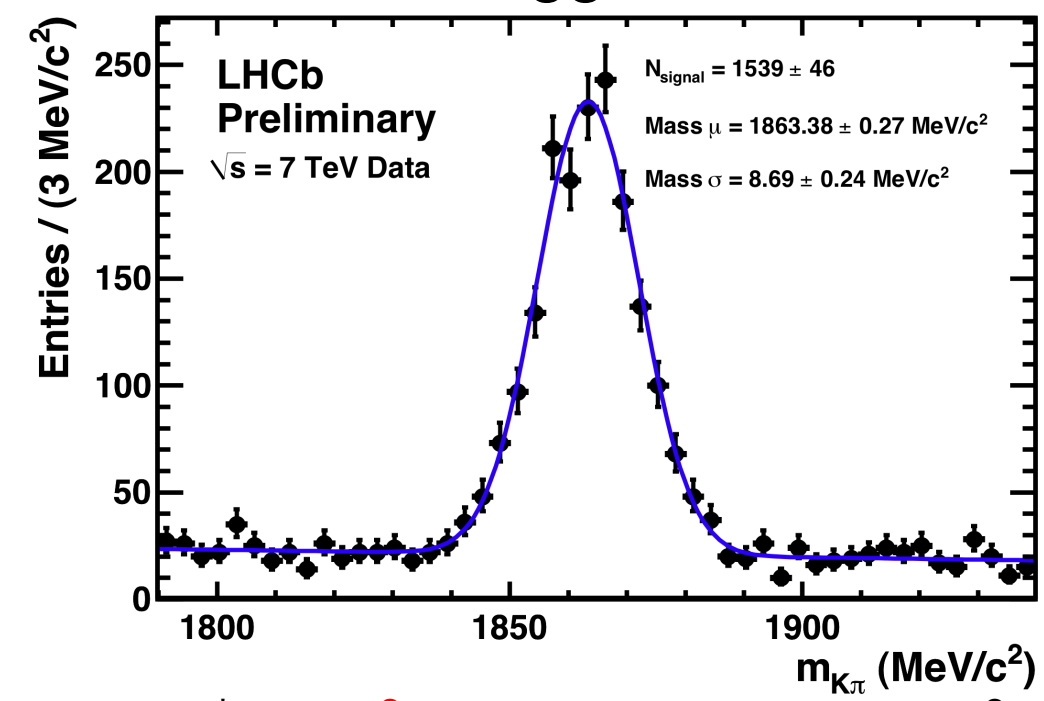

$\mathrm{D}^{*+} \rightarrow \mathrm{D}^{0}(\rightarrow \mathrm{K} \pi) \pi^{+}\left[\right.$tagged $\left.\mathrm{D}^{0}\right]$

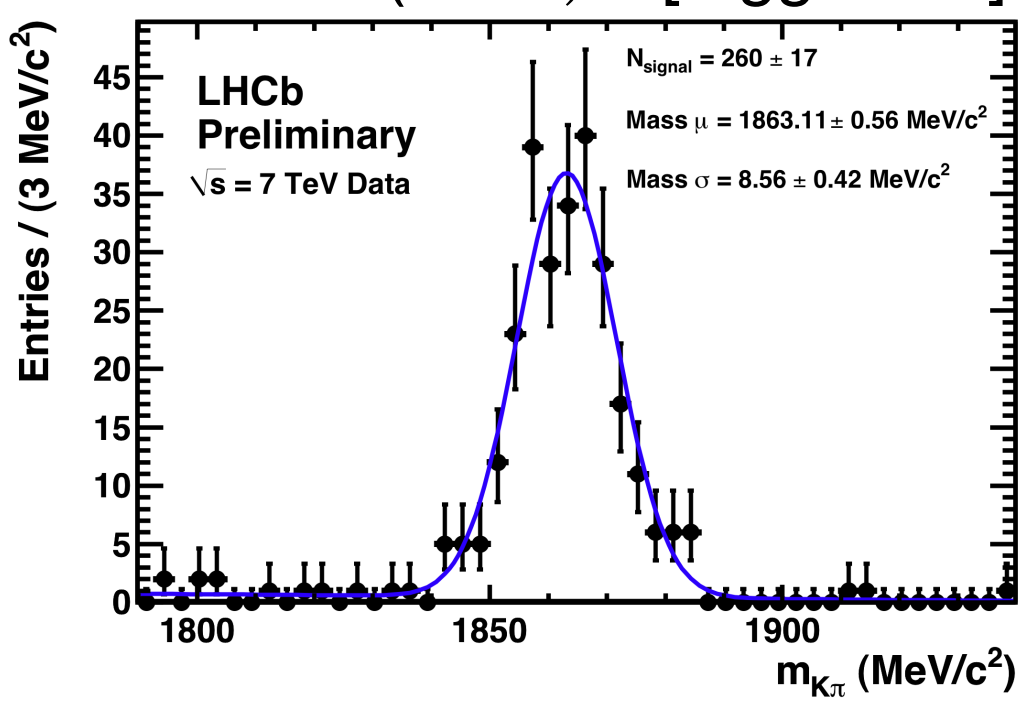

Expected several million of untagged and tagged $D^{0} \rightarrow K \pi @ 0.1 \mathrm{fb}^{-1}$

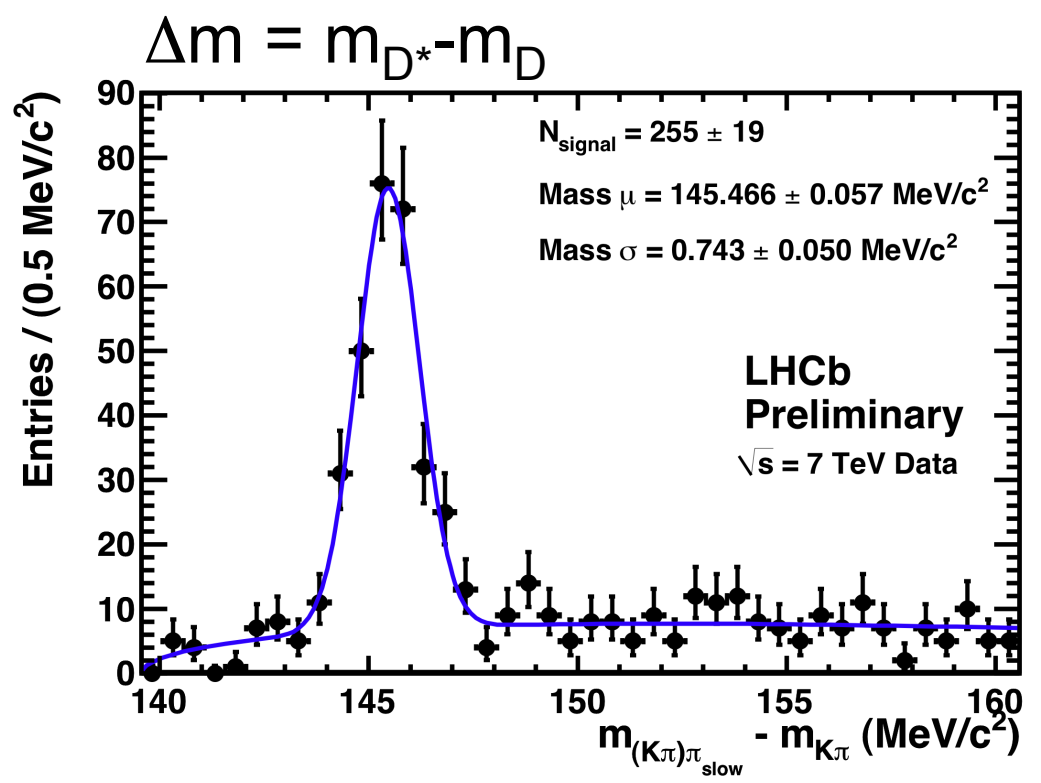

$m_{D^{*}}-m_{D}<\mu_{\Delta m} \pm 2 \sigma_{\Delta m}$ 


\section{$D^{0}$ in LHCb @ 800ub-1}

\section{$\mathrm{D}^{0} \rightarrow \mathrm{KK}$ untagged}
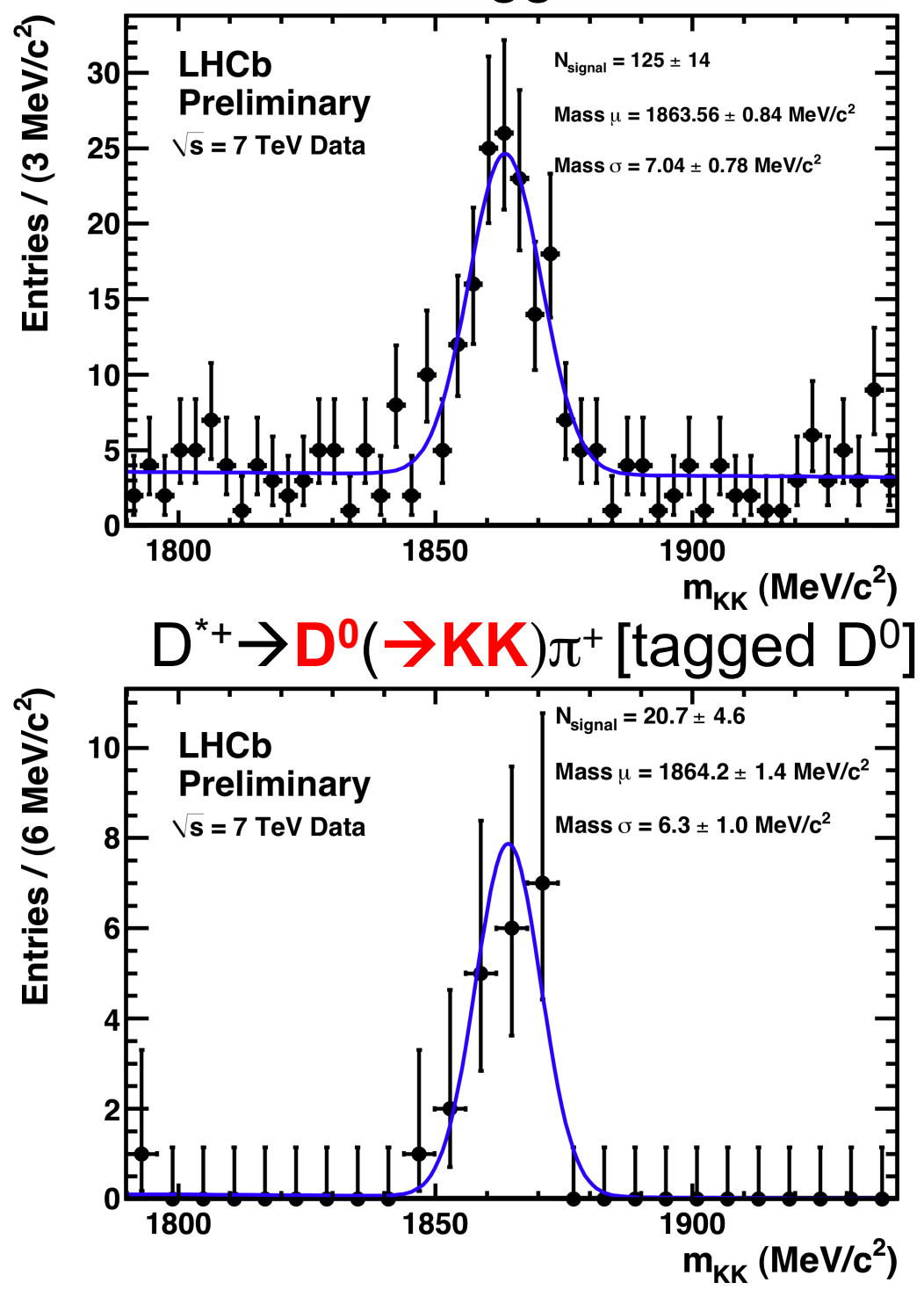

Expected several million of untagged and tagged $\mathrm{D}^{0} \rightarrow \mathrm{KK} @ 0.1 \mathrm{fb}^{-1}$

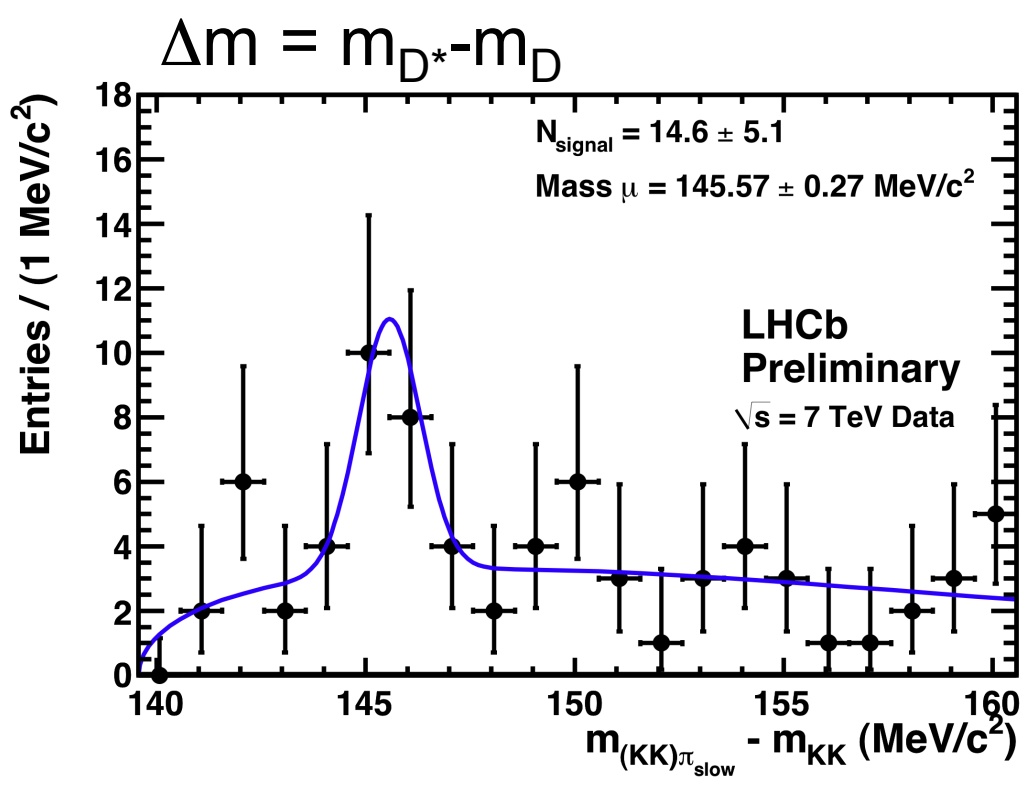




\section{Direct CPV in charm}
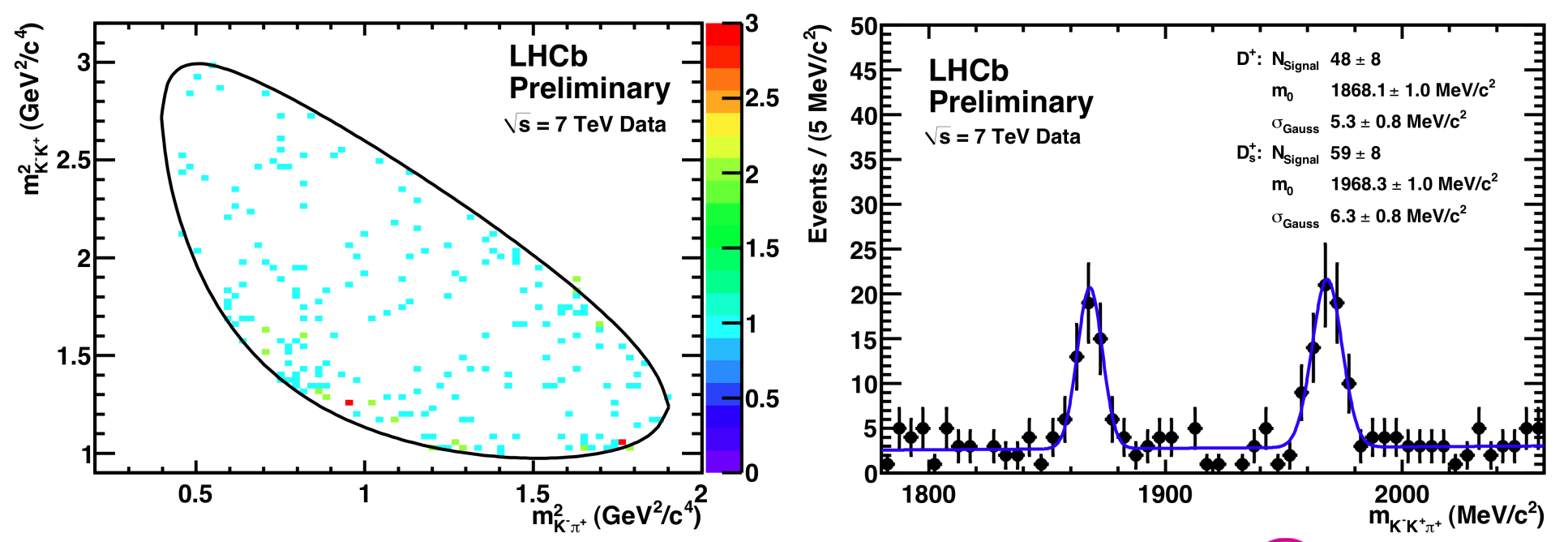
$\mathrm{D}_{(\mathrm{s})}^{+} \rightarrow \mathrm{K}^{+} \mathrm{K}^{-} \pi^{+}$
@ $800 \mu b^{-1}$
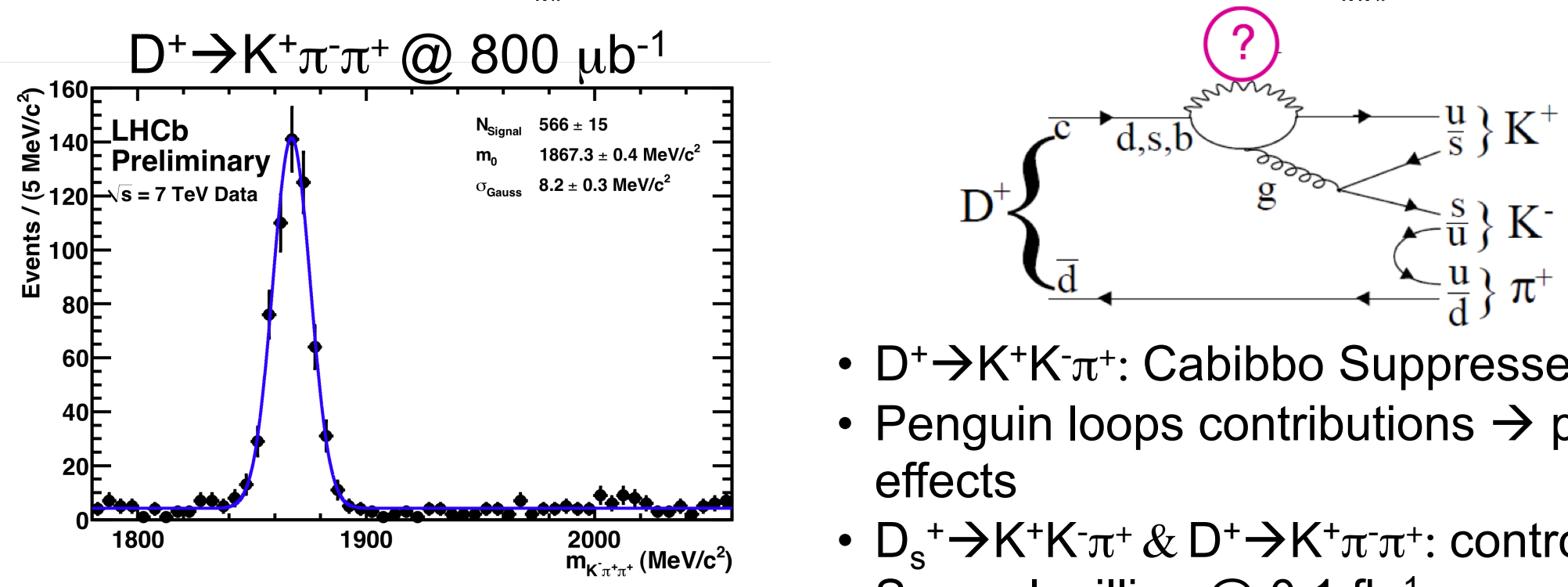

- $\mathrm{D}^{+} \rightarrow \mathrm{K}^{+} \mathrm{K}^{-} \pi^{+}$: Cabibbo Suppressed decay

- Penguin loops contributions $\rightarrow$ possible NP effects

- $\mathrm{D}_{\mathrm{s}}^{+} \rightarrow \mathrm{K}^{+} \mathrm{K}^{-} \pi^{+} \& \mathrm{D}^{+} \rightarrow \mathrm{K}^{+} \pi^{-} \pi^{+}$: control channels

- Several million @ $0.1 \mathrm{fb}^{-1}$ 


\section{What about $B$ decays?}

\section{LHCb}

$\mathrm{B}^{+} \rightarrow \mathrm{J} / \Psi \mathrm{K}^{+}$

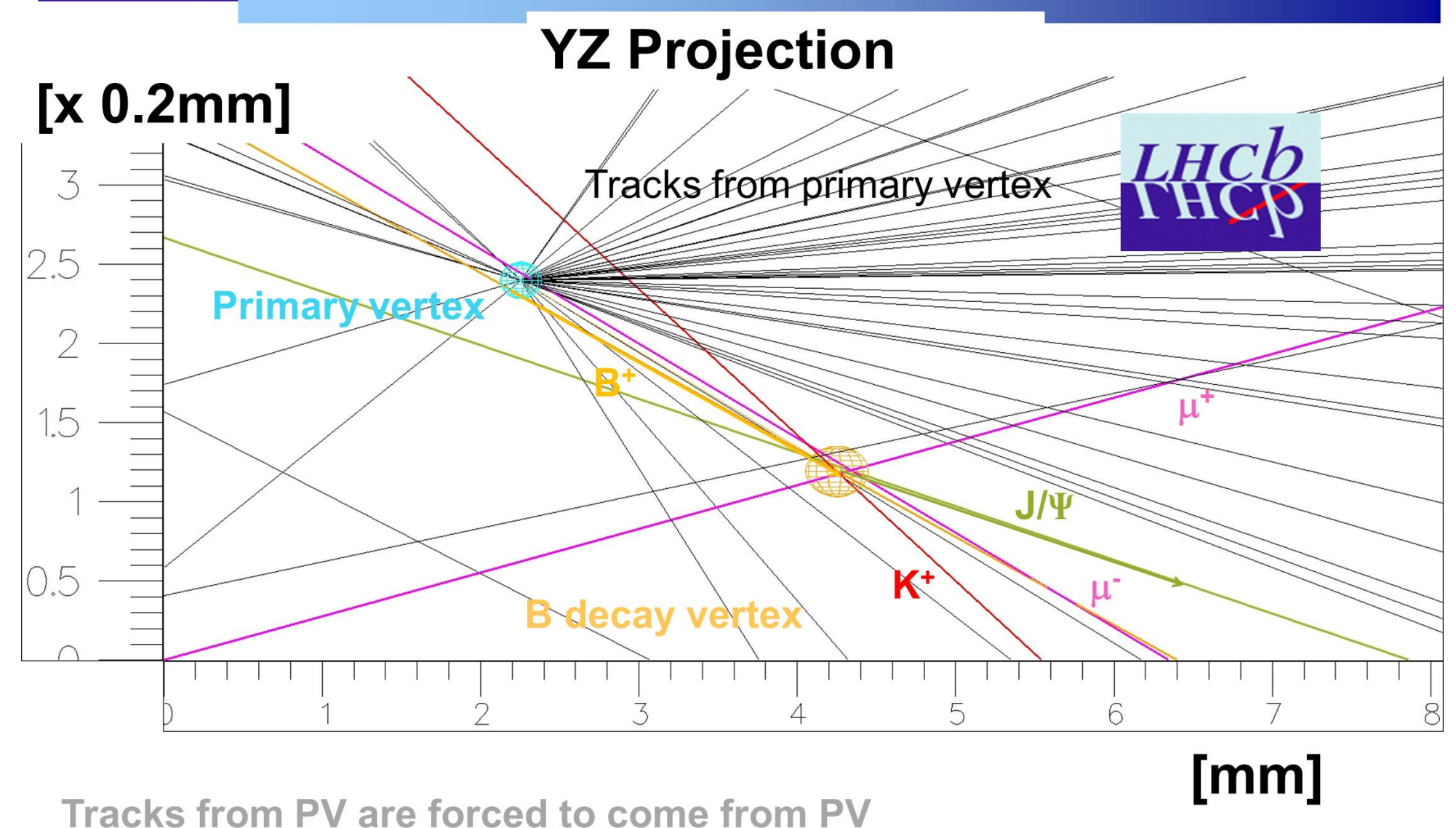




\section{$\gamma$ from trees}

$\mathrm{B}^{-} \longrightarrow \mathrm{D}^{0} \mathrm{~K}^{-}$

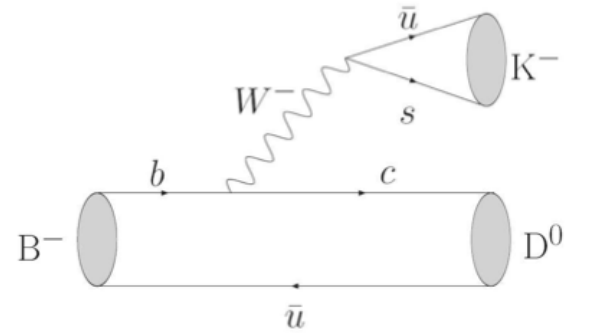

$\mathrm{B}^{-}$ $\overline{\mathrm{D}}^{0} \mathrm{~K}^{-}$

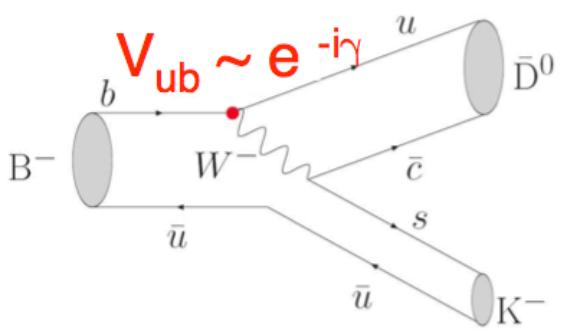

Many parameters involved can dilute sensibility to $\gamma$

$\mathbf{R}_{\mathrm{CP}} \sim 2 \mathrm{r}_{\mathrm{B}} \cos \left(\delta_{\mathrm{B}}\right) \cos (\mathrm{Y})$

$A_{C P} \sim 2 r_{B} \sin \left(\delta_{B}\right) \sin (y) / R_{C P}$

Need to over constrain the system with various

channels

1. GLW (Gronau, London, Wyler):

- Exploit asymmetries for $\mathrm{D}^{0}$ decaying to CP-even or CP-odd eigenstates

- $\mathrm{D}^{0} \rightarrow \mathrm{KK}, \mathrm{D}^{0} \rightarrow \pi \pi$

- Also $\mathrm{B}^{0} \rightarrow \mathrm{D}^{0}(\rightarrow \mathrm{hh}) \mathrm{K}^{*}$

2. ADS (Atwood, Dunietz, Soni):

- Exploit asymmetries for $D^{0}$ to flavor specific channel $\left(D^{0} \rightarrow K \pi, D^{0} \rightarrow K \pi \pi \pi\right)$

3. Dalitz analysis (Giri, Grossman, Soffer and Zupan):

- Exploit asymmetries in $\mathrm{D}^{0} \rightarrow \mathrm{K}_{S}{ }^{0} \pi \pi$ and $\mathrm{D}^{0} \rightarrow \mathrm{K}_{S}{ }^{0} \mathrm{KK}$

$$
\text { - } \sigma_{\mathrm{LHCb}} \sim 7^{\circ} @ 1 \mathrm{fb}^{-1}
$$




\section{$B_{s}$ mixing phase}

- Very precisely predicted in SM $\rightarrow \beta_{\mathrm{s}}=0.02$

- Tevatron measurements give us a hint for NP

- Golden channel: $B_{s} \rightarrow J / \Psi \Phi$

- Penguin pollution negligible

- $\mathrm{J} / \Psi \rightarrow \mu \mu$ : very clean signal for LHCb

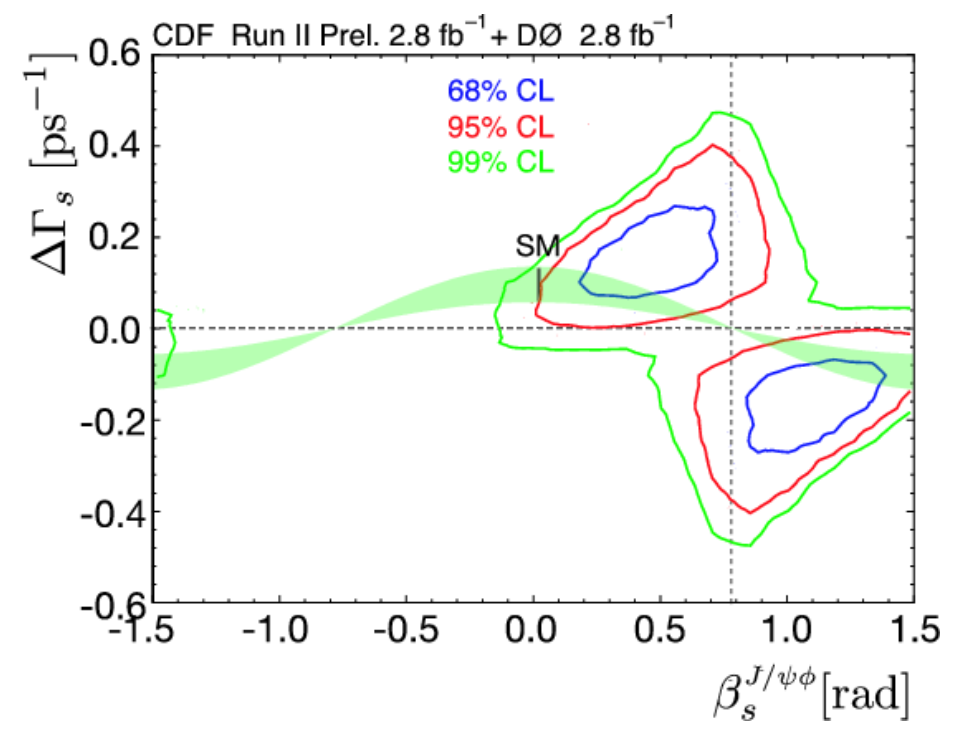

History:

Old result: $\beta_{\mathrm{s}}=[0.10,1.42] @ 95 \%$ C.L. New result: $\beta_{\mathrm{s}}=[-0.1,0.7] @ 95 \%$ C.L.
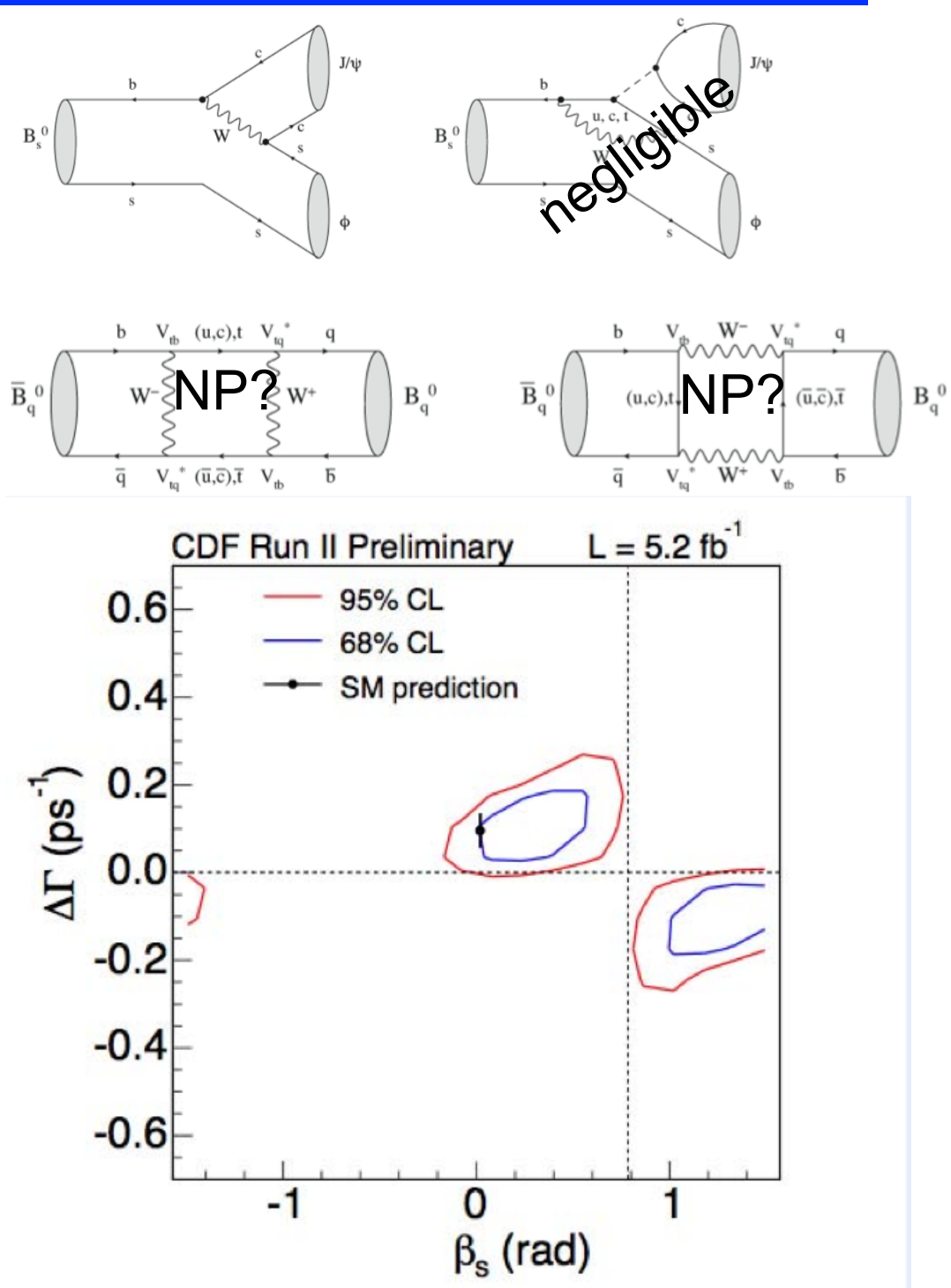


\section{$\mathrm{B}_{\mathrm{s}}$ mixing phase}

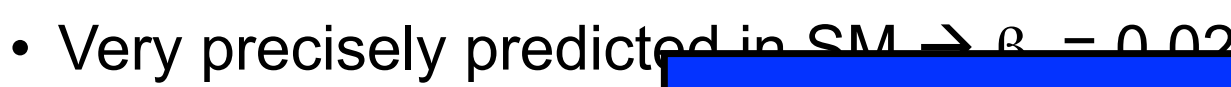

- Tevatron measuremer LHCb has in any case a

- Golden channel: $\mathrm{B}_{\mathrm{s}} \rightarrow$

- Pengu

- $\mathrm{J} / \Psi \rightarrow$

0.6
-0.2
-0.4
-0.6


Intrigui
Old res
New re
Nen

\section{great potential}

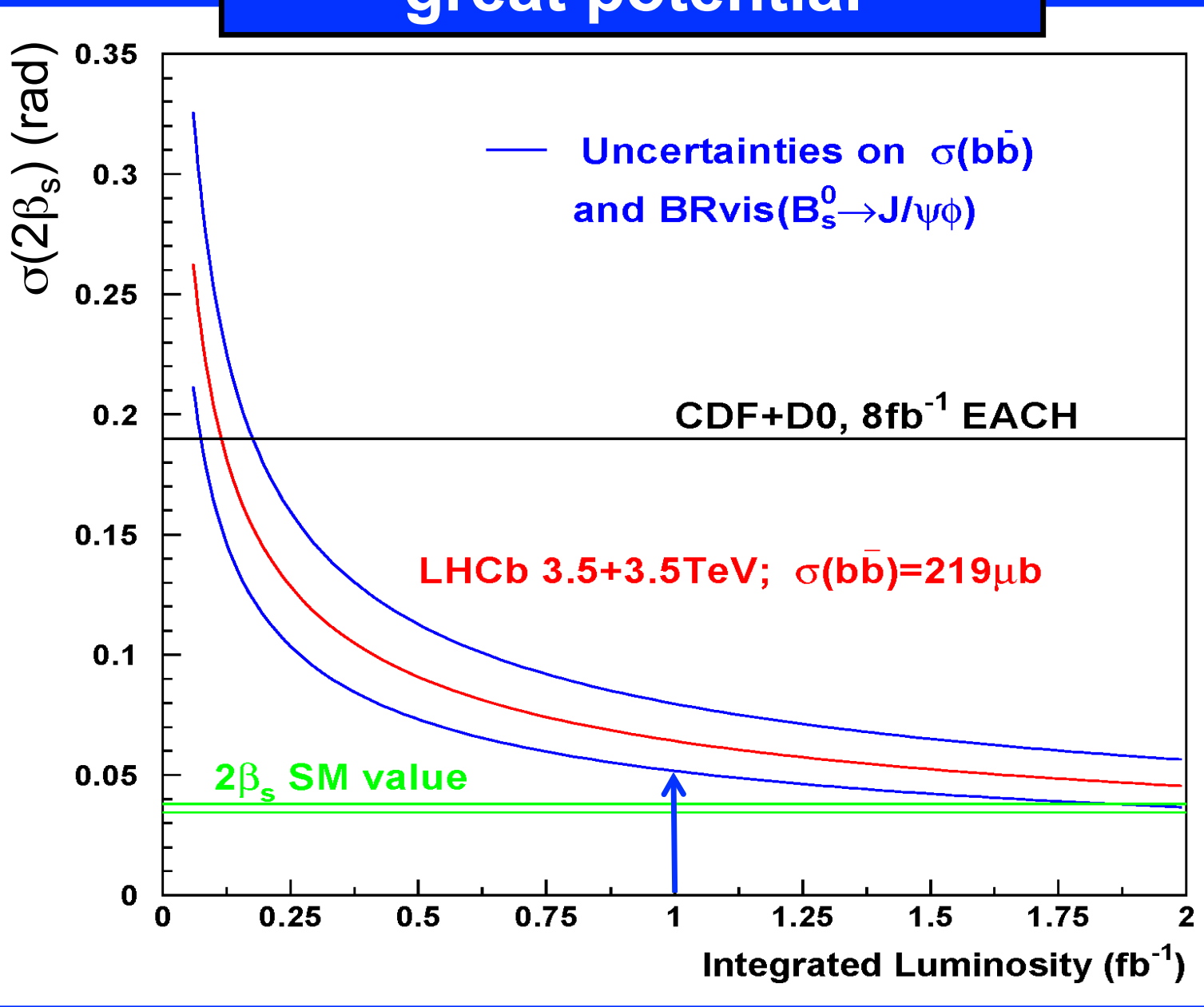

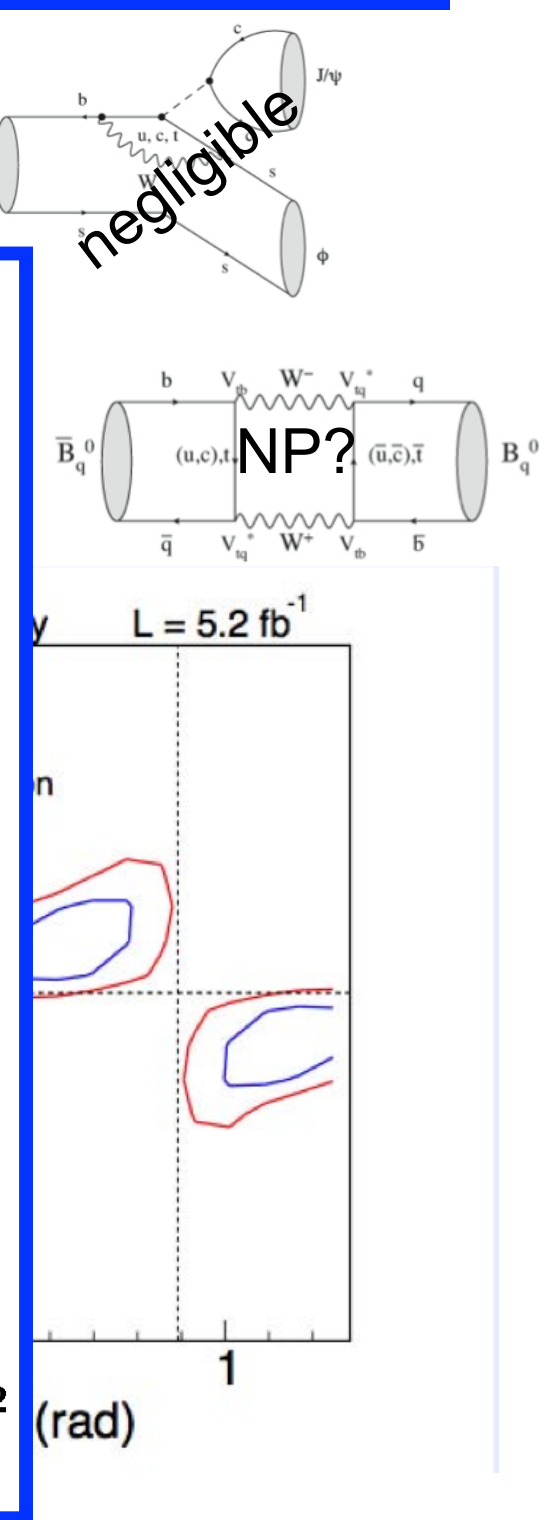

19 


\section{$\mathrm{B}_{\mathrm{s}} \rightarrow \mu \mu(\mathrm{I})$}

- Flavor Changing Neutral Current process

- $\operatorname{BR}\left(B_{s} \rightarrow \mu \mu\right)=(3.35 \pm 0.32) \times 10^{-9}$ [Blanke et al., JHEP 0610:003,2006]

- Very sensible to NP $\rightarrow$ BR altered from SM prediction
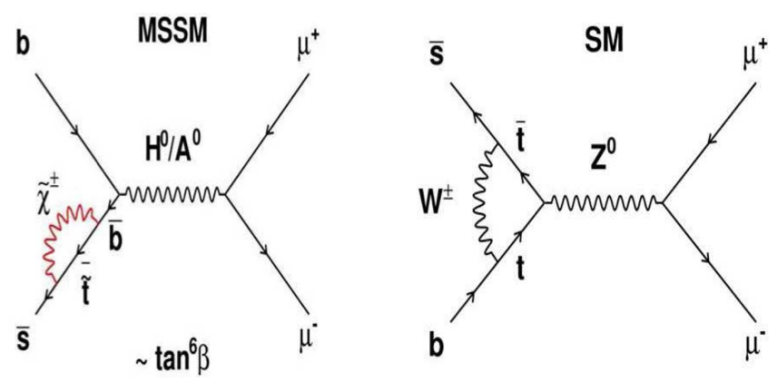

arXiv:0709.0098 [hep- ph]
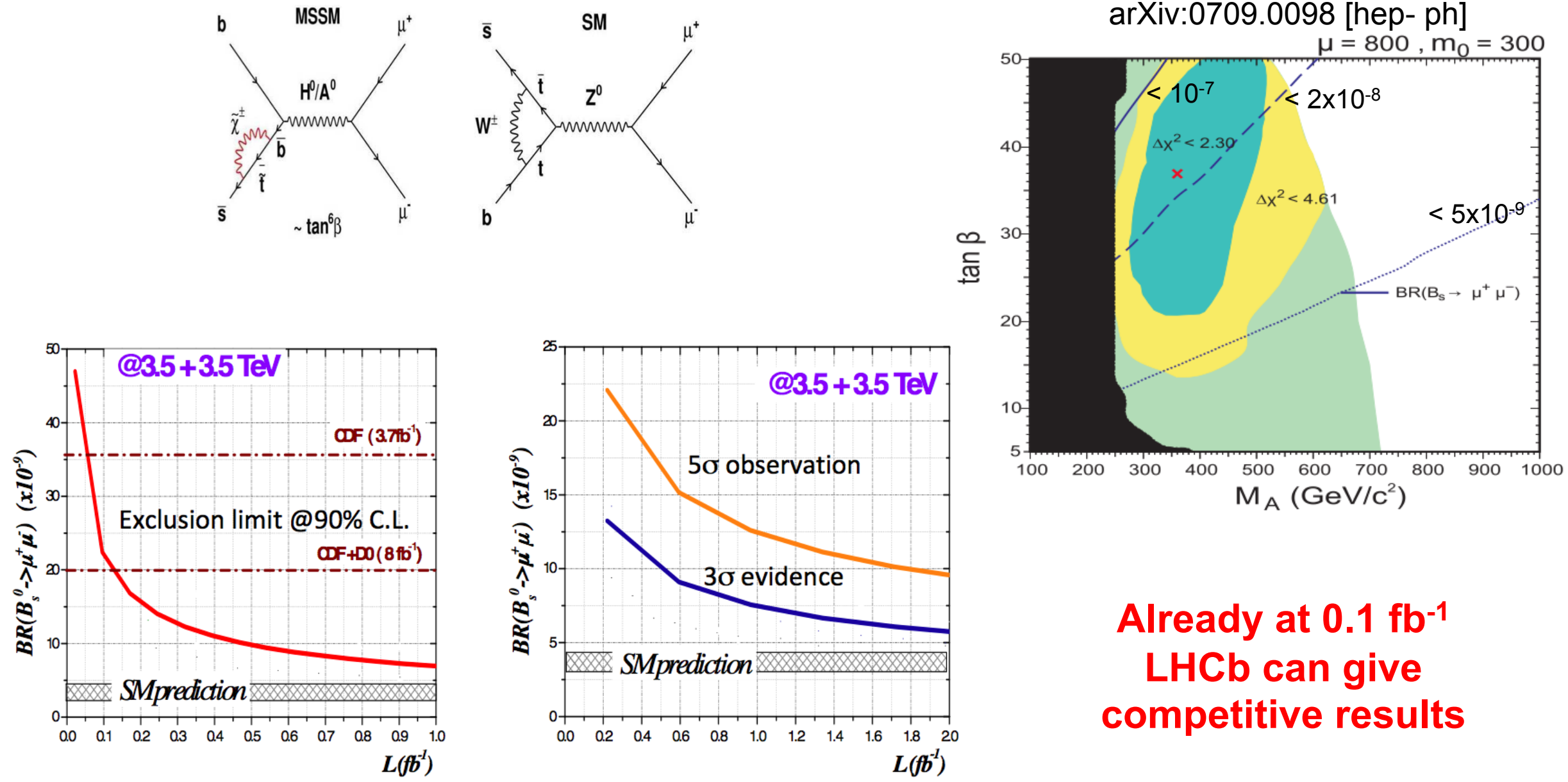

Already at $0.1 \mathrm{fb}^{-1}$ LHCb can give competitive results 


\section{$\mathrm{B}_{\mathrm{s}} \rightarrow \mu \mu(\mathrm{II})$}

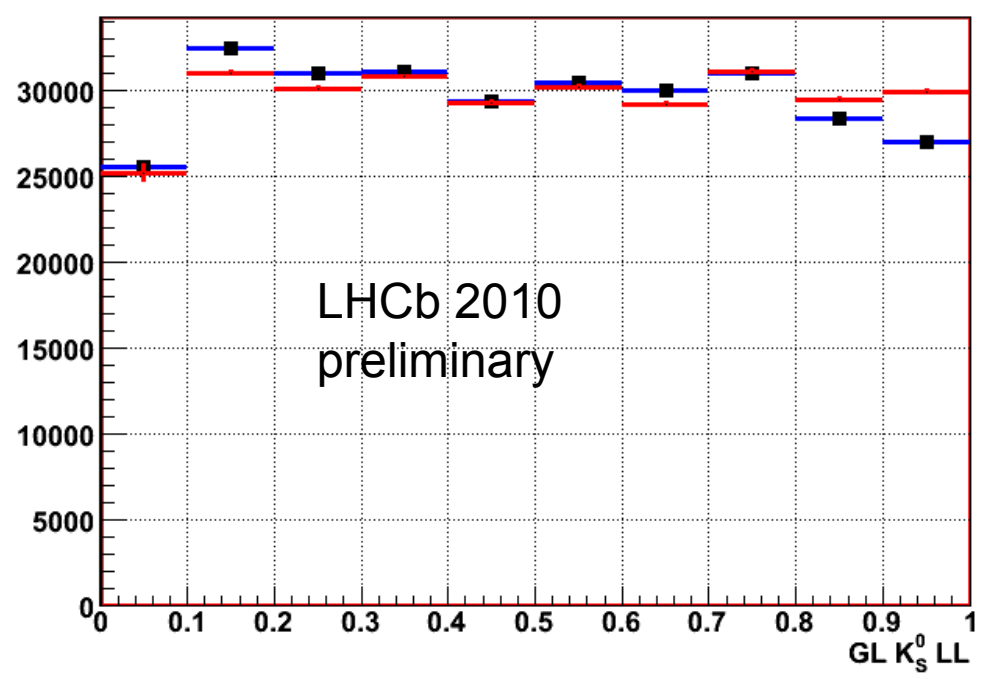

- Strategy (as at Tevatron):

- Very loose selection

- Loooking for signal using a global likelihood method

- Calibration already started using $\mathrm{K}_{\mathrm{s}}{ }^{0} \rightarrow \pi^{+} \pi^{-}$and $\mathrm{J} / \Psi \rightarrow \mu^{+} \mu^{-}$
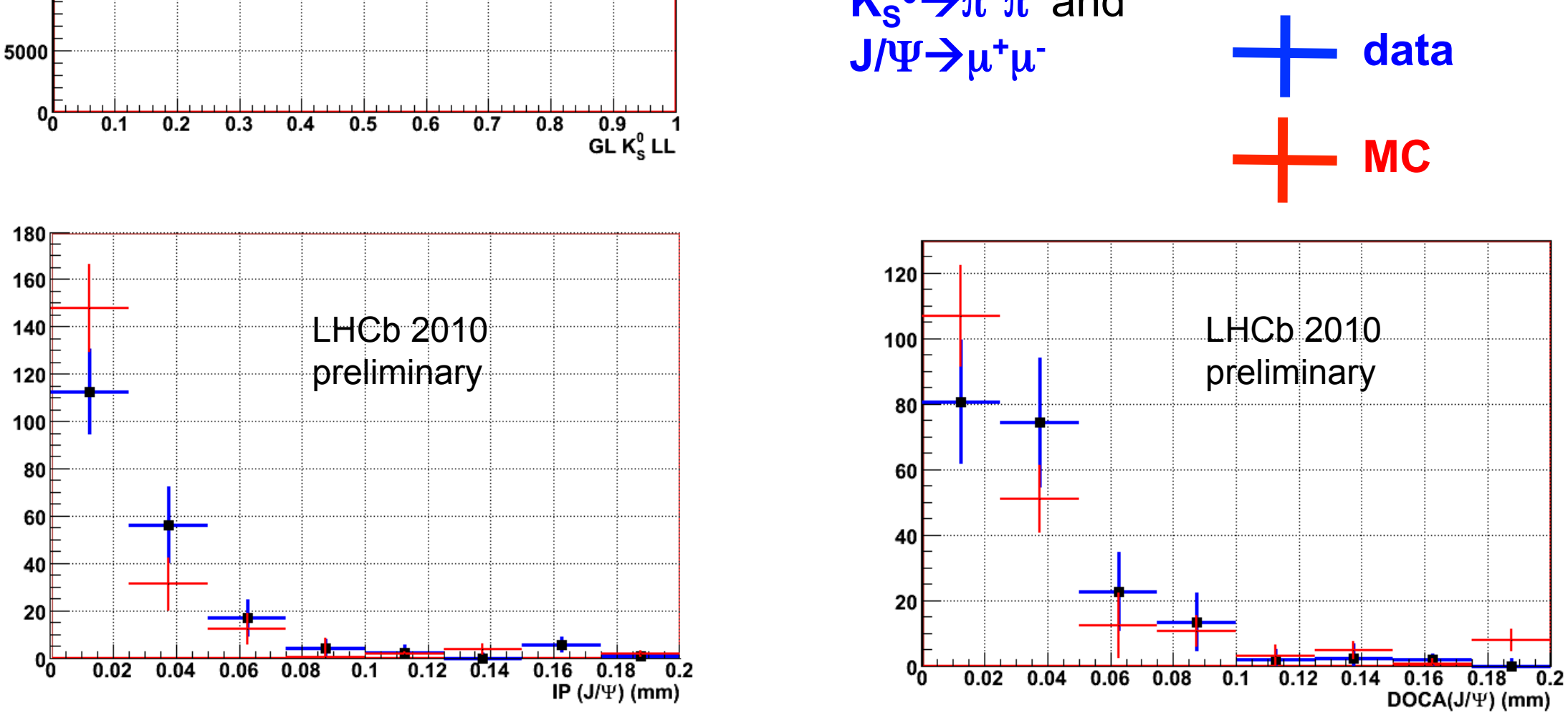


\section{$\mathrm{B}_{\mathrm{s}} \rightarrow \mu \mu(\mathrm{II})$}

- Trigger must to be as efficient as possible:

- Calibration with $\mathrm{J} / \Psi \rightarrow \mu^{+} \mu^{-}$

data

$+\mathrm{MC}$
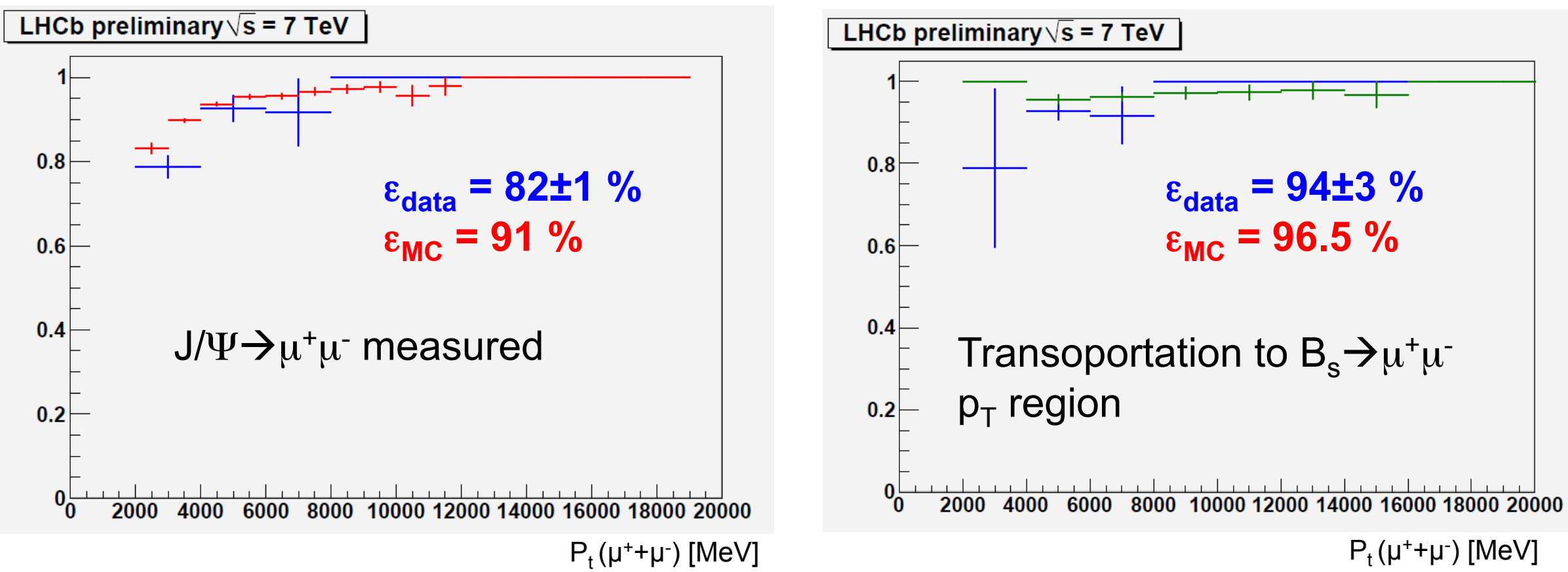


\section{$\mathrm{B}_{\mathrm{s}} \rightarrow \mu \mu(\mathrm{III})$}

- Importance of MuonID:

- Mis-ID: using $\mathrm{K}_{S}{ }^{0}$ and $\Lambda$ as source of $\pi$ and $p$

- MuonID: using $\mathrm{J} / \Psi \rightarrow \mu^{+} \mu^{-}$
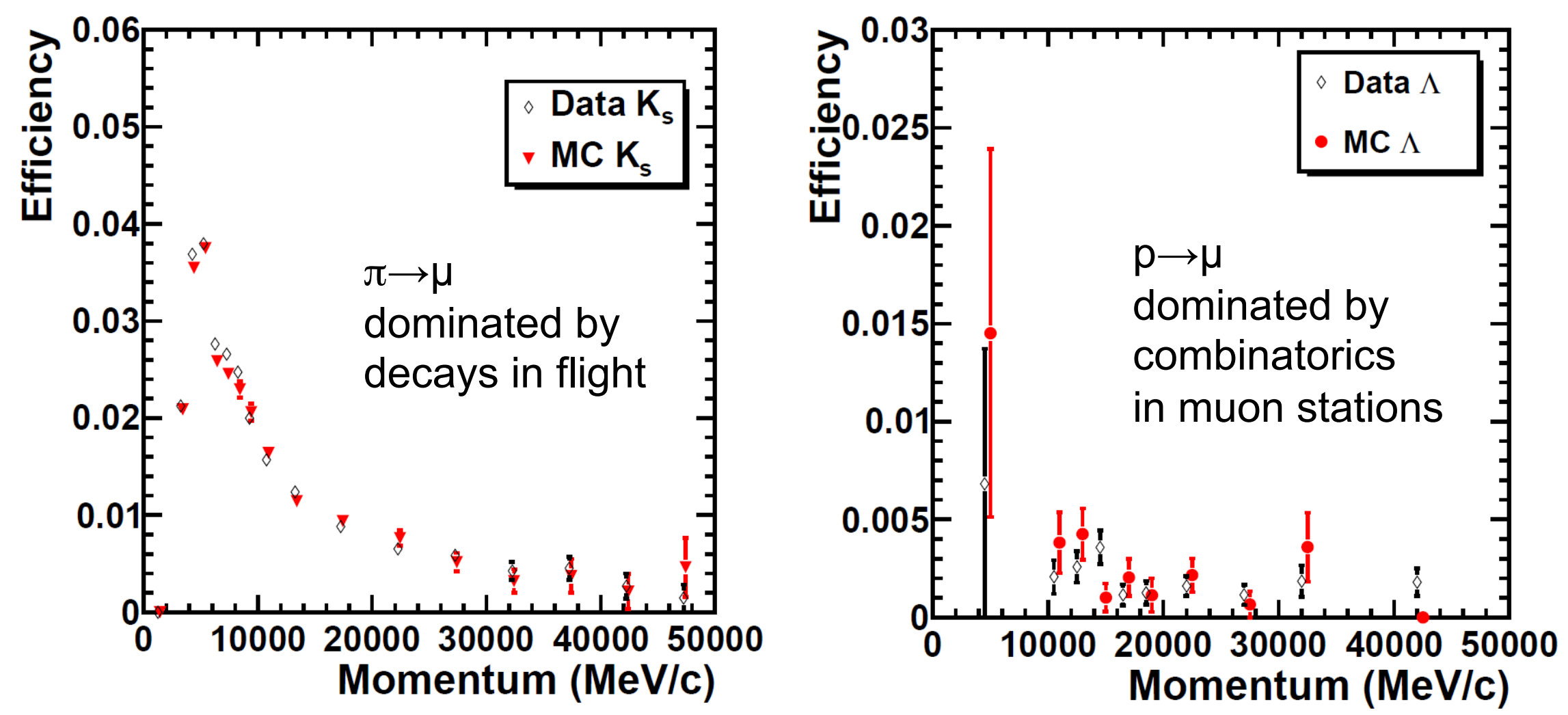


\section{$\mathrm{B}^{0} \rightarrow \mathrm{K}^{*} \mu^{+} \mu^{-}(\mathrm{I})$}
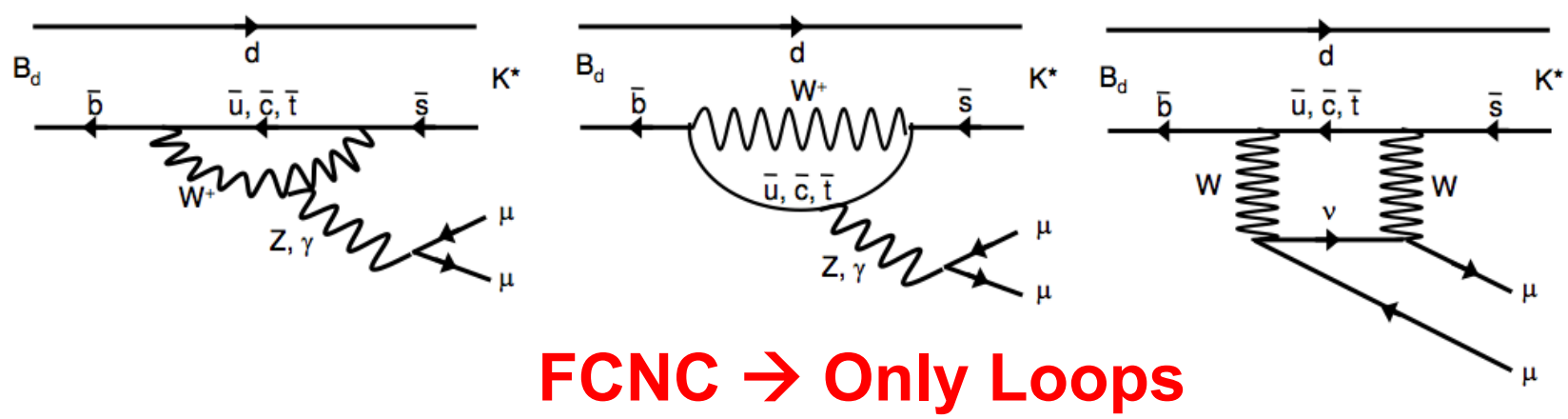

Decay totally described via geometrical observables

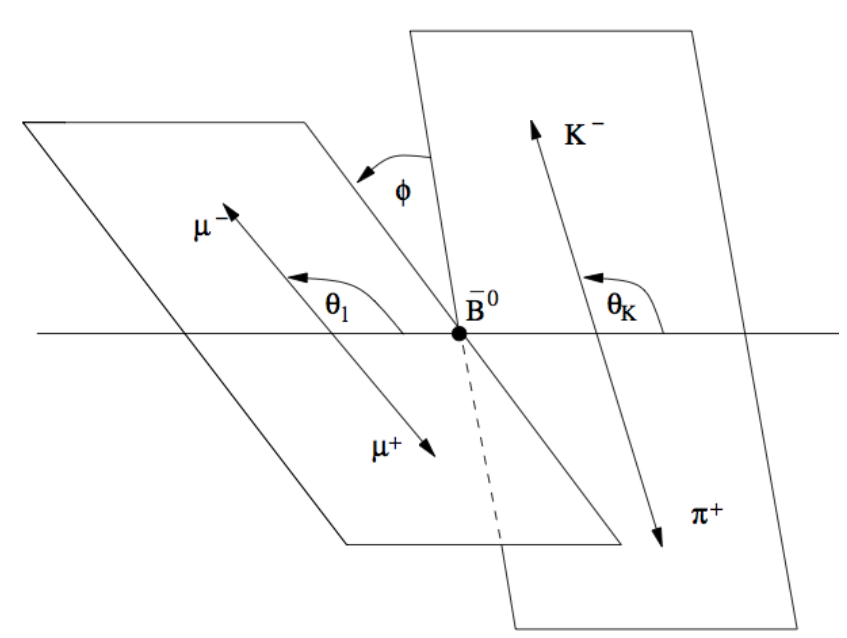

FCNC $\rightarrow$ Only Loops

NP

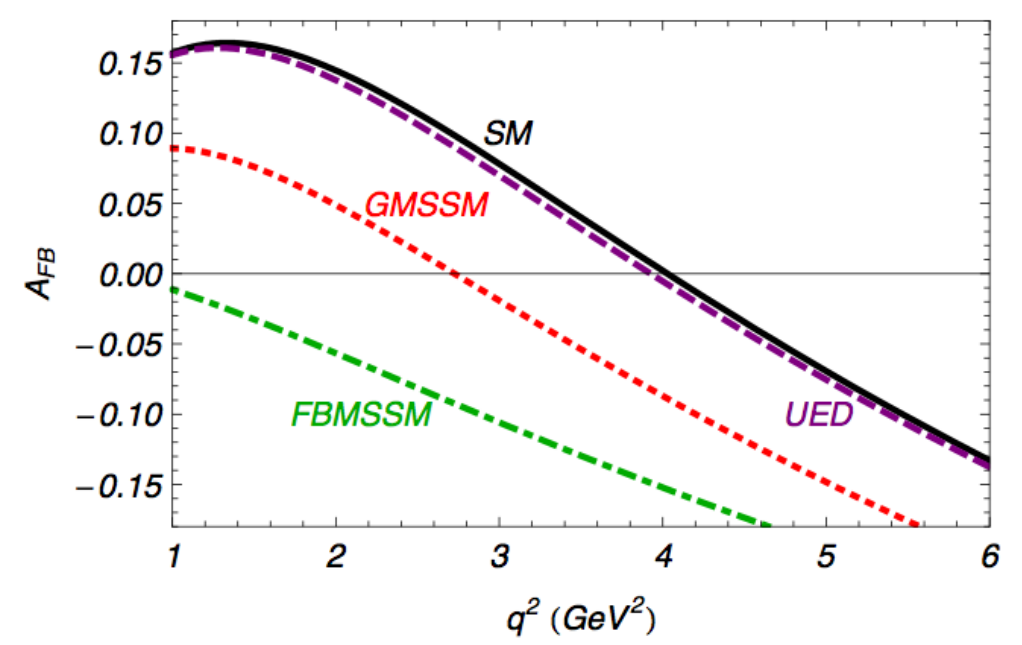

The point at $A_{F B}=0$ can probe various NP models 


\section{$\mathrm{B}^{0} \rightarrow \mathrm{K}^{*} \mu^{+} \mu^{-}$(II)}
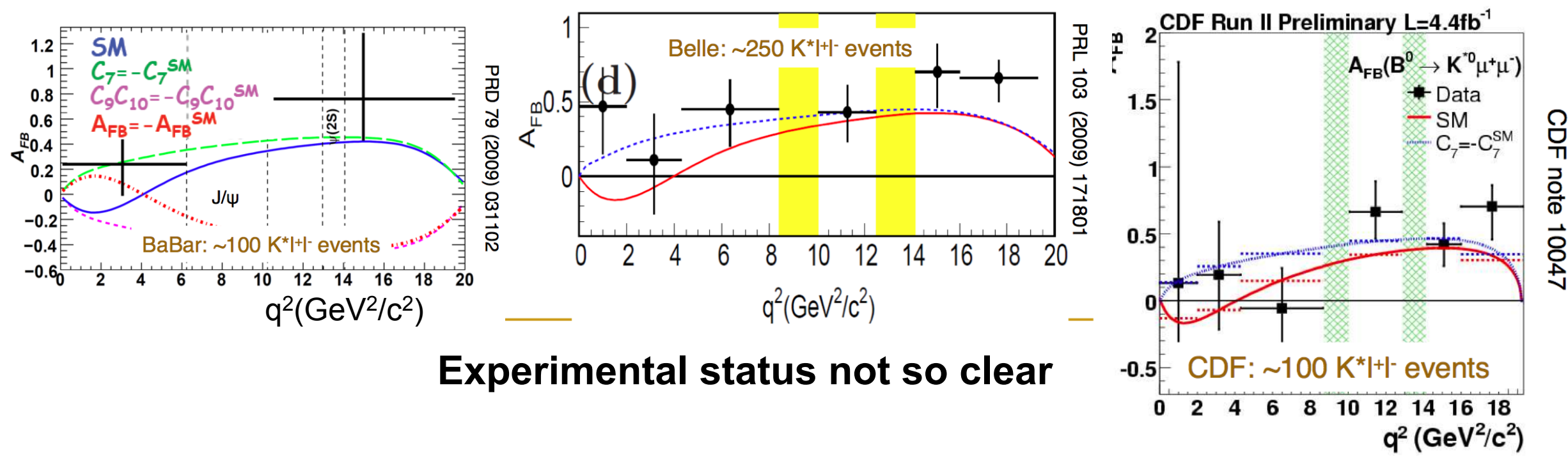

\section{LHCb @ 1 fb $^{-1}$ -1200 events}

If NP signal not so large will be necessary to more bins in $q^{2}$ $\sigma\left(\mathrm{q}^{2}\right) \sim 0.8 \mathrm{GeV}^{2} @ 1 \mathrm{fb}^{-1}$

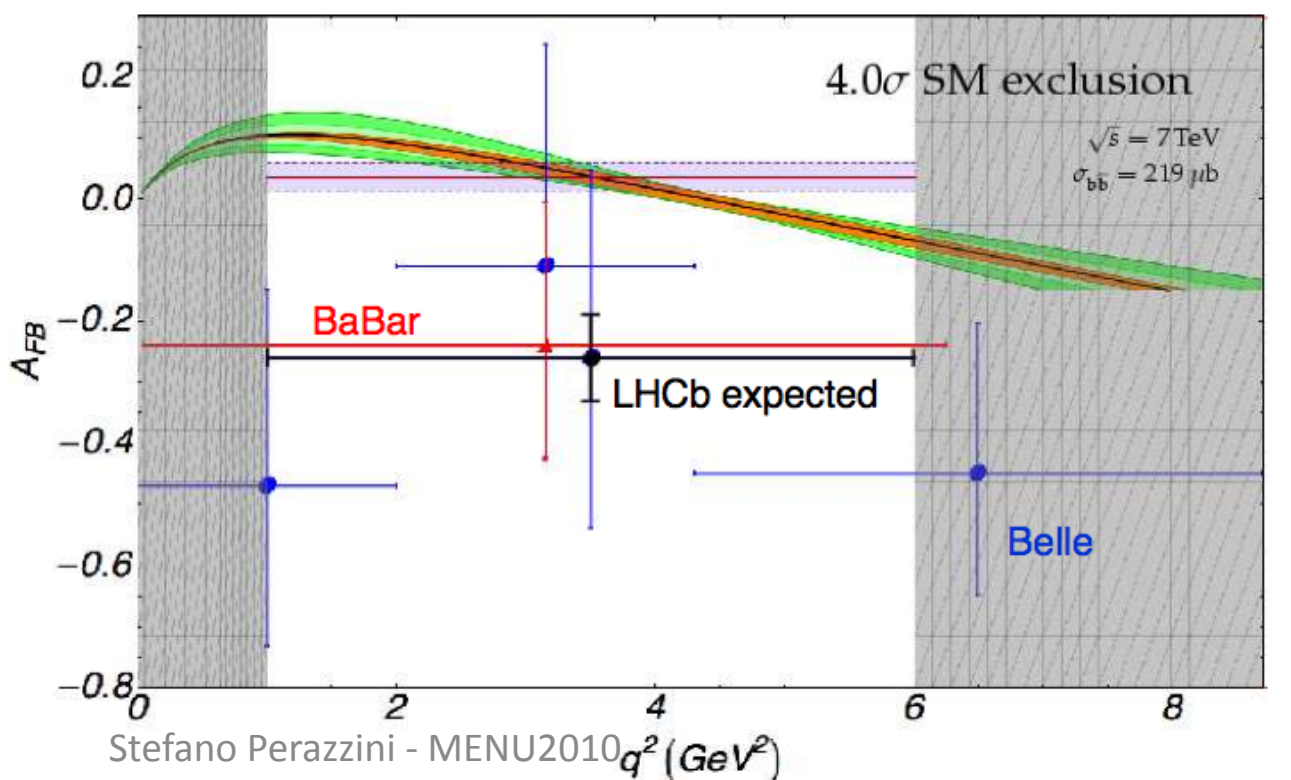




\section{No time to mention}

- $\mathrm{V}^{0}$ analysis:

- $\Lambda$, anti- $\Lambda$ and $\mathrm{K}_{\mathrm{s}}{ }^{0}$ production

- $(\Lambda$ and anti- $\Lambda) / \mathrm{K}_{\mathrm{s}}^{0}$ ratio

- $\mathrm{CP}$ asymmetries in gluonic $\mathrm{b} \rightarrow \mathrm{s}$ penguin decays

- $\mathrm{B}_{\mathrm{s}} \rightarrow \phi \phi, \mathrm{K}^{*} \mathrm{~K}^{*}$

- Charmless hadronic 2-body and 3-body B Decays

- Radiative penguin decays

- $\mathrm{B}_{\mathrm{s}} \rightarrow \phi \mathrm{Y}, \mathrm{B} \rightarrow \mathrm{K}^{*} \mathrm{Y}$

- More CKM metrology

$-\sin 2 \beta\left(\mathrm{B}_{\mathrm{d}} \rightarrow \mathrm{J} / \psi \mathrm{K}_{\mathrm{s}}\right)$

$-\alpha(B \rightarrow \rho \pi)$ 


\section{Conclusions}

1. LHC has started:

a) The lower centre-of-mass energy of LHC loss will not affect dramatically LHCb performances $\rightarrow$ wait for a measurement of $\sigma_{b b}$

b) Luminosity conditions opened a very promising window on charm and is expected to approach the LHCb design luminosity in 2011

2. LHCb status is good:

a) Already seen a lot of well known strange and charm peaks

b) Calibration well underway (alignment and PID)

c) First B candidates observed

3. Physics programme:

a) Charm analysis will exploit several million of $D$ meson decays

b) B physics core analyses can lead to competitive measurements already with $0.1 \mathrm{fb}^{-1}$

c) $1 \mathrm{fb}^{-1}$ should lead to quite exciting results and New Physics 